\title{
TISSUE ENGINEERING SCALED-UP, ANATOMICALLY SHAPED OSTEOCHONDRAL CONSTRUCTS FOR JOINT RESURFACING
}

\author{
T. Mesallati ${ }^{1,2}$, E.J. Sheehy ${ }^{1,2}$, T. Vinardell ${ }^{3}$, C.T. Buckley ${ }^{1,2}$ and D.J. Kelly ${ }^{1,2,4,5 *}$ \\ ${ }^{1}$ Trinity Centre for Bioengineering, Trinity Biomedical Sciences Institute, Trinity College Dublin, Dublin, Ireland. \\ ${ }^{2}$ Department of Mechanical and Manufacturing Engineering, School of Engineering, Trinity College Dublin, \\ Dublin, Ireland. \\ ${ }^{3}$ School of Agriculture and Food Science, University College Dublin, Belfield, Dublin 4, Ireland. \\ ${ }^{4}$ Department of Anatomy, Royal College of Surgeons in Ireland, 123 St. Stephen's Green, Dublin 2, Dublin, Ireland. \\ ${ }^{5}$ Advanced Materials and Bioengineering Research Centre (AMBER), \\ Royal College of Surgeons in Ireland and Trinity College Dublin, Dublin, Ireland.
}

\begin{abstract}
Arthroplasty is currently the only surgical procedure available to restore joint function following articular cartilage and bone degeneration associated with diseases such as osteoarthritis (OA). A potential alternative to this procedure would be to tissue-engineer a biological implant and use it to replace the entire diseased joint. The objective of this study was therefore to tissue-engineer a scaled-up, anatomically shaped, osteochondral construct suitable for partial or total resurfacing of a diseased joint. To this end it was first demonstrated that a bone marrow derived mesenchymal stem cell seeded alginate hydrogel could support endochondral bone formation in vivo within the osseous component of an osteochondral construct, and furthermore, that a phenotypically stable layer of articular cartilage could be engineered over this bony tissue using a co-culture of chondrocytes and mesenchymal stem cells. Co-culture was found to enhance the in vitro development of the chondral phase of the engineered graft and to dramatically reduce its mineralisation in vivo. In the final part of the study, tissue-engineered grafts $(\sim 2 \mathrm{~cm}$ diameter) mimicking the geometry of medial femorotibial joint prostheses were generated using laser scanning and rapid prototyped moulds. After 8 weeks in vivo, a layer of cartilage remained on the surface of these scaled-up engineered implants, with evidence of mineralisation and bone development in the underlying osseous region of the graft. These findings open up the possibility of a tissueengineered treatment option for diseases such as OA.
\end{abstract}

Keywords: Alginate, Bone tissue-engineering, Cartilage tissue-engineering, Co-culture, Rapid prototyping, Selfassembly, Endochondral.

\footnotetext{
*Address for correspondence:

Daniel J. Kelly

Trinity Centre for Bioengineering

School of Engineering

Trinity College Dublin, Ireland
}

Telephone number: +353-1-896-3947

Fax number: +353-1-679-5554

E-mail: kellyd9@tcd.ie

\section{Introduction}

Osteoarthritis (OA), the most common form of arthritis, is a degenerative disease of the joints affecting millions of people worldwide (Woolf and Pfleger, 2003). The disease is characterised by joint pain and dysfunction resulting from the progressive loss of articular cartilage within synovial joints. Prevalence of the disease is expected to increase by $40 \%$ by 2025 (Hayes et al., 2012). Treatment options for OA are limited to surgical replacement of the diseased joint with a metal and polyethylene prosthesis (Guilak, 2010). While this procedure is well established, it is not without its limitations and failures are not uncommon (Ma et al., 2005; Pavone et al., 2001; Seil and Pape, 2011). Joint replacement prostheses also have a finite lifespan, making them unsuitable for the growing population of younger and more active patients requiring treatment for OA (Guilak, 2010; Keeney et al., 2011; Kurtz et al., 2009). In recent years, there has been increased interest in the use of cell- and tissue-engineering based therapies for the treatment of focal cartilage defects (Brittberg et al., 1994; Temenoff and Mikos, 2000). While significant progress has been made in this field, realising an efficacious therapeutic option for the treatment of OA remains elusive and is considered to be one of the greatest challenges in the field of orthopaedic medicine. Given that OA affects multiple tissues in the diseased joint, including the articular cartilage and underlying subchondral bone, the goal of this study was to tissue-engineer a scaled-up, anatomically shaped osteochondral construct suitable for partial or total joint resurfacing.

A number of different strategies have been developed to engineer osteochondral constructs. These include the development of bi-phasic or multi-layered scaffolds (Mano and Reis, 2007; Martin et al., 2007; Rodrigues et al., 2012; Sheehy et al., 2013), physical conditioning of tissues through the use of novel bioreactors (Grayson et al., 2010; Wang et al., 2004; Wendt et al., 2005) and spatial growth factor or gene delivery systems (Chen et al., 2011; Mason et al., 1998; Santo et al., 2013). It has also been possible to engineer scaffolds and grafts mimicking the geometrical form of articular surfaces (Alhadlaq et al., 2004; Ding et al., 2013; Hung et al., 2003; Lee et al., 2010; Lee et al., 2009). However, tissue-engineering anatomically accurate osteochondral constructs of scale remains a significant challenge in the field. An alternative strategy to engineering osseous tissues of scale, is to leverage the inherent tendency of chondrogenically primed bone marrowderived mesenchymal stem cells (BMSCs) to become 
hypertrophic and undergo endochondral ossification in vivo (Farrell et al., 2011; Farrell et al., 2009; Janicki et al., 2010; Scotti et al., 2013; Scotti et al., 2010). While this is a major limitation associated with MSCs for articular cartilage tissue-engineering, this property has recently been leveraged for large bone defect regeneration (Harada et al., 2014; van der Stok et al., 2014). Previous studies have demonstrated that cartilaginous constructs engineered using BMSCs embedded in a hydrogel will proceed along an endochondral pathway in vivo (Dickhut et al., 2008; Vinardell et al., 2012). Indeed, previous work in our lab has shown that it is possible to engineer osteochondral constructs by spatially regulating endochondral ossification within bi-layered agarose cartilaginous grafts (Sheehy et al., 2013). As agarose does not degrade in vivo and hence impedes vascularisation and the conversion of engineered calcified cartilage into functional bone (Sheehy et al., 2014), the first objective of this study was to investigate if a BMSC seeded alginate hydrogel (as opposed to agarose) could support endochondral bone formation within an osteochondral construct. This was motivated by the fact that alginate is commonly used for in vitro cartilage tissue-engineering purposes (Igarashi et al., 2010; Lee and Mooney, 2012; Shen et al., 2009), and furthermore, has been combined with MSCs and/or different growth factors for use in bone regeneration (Kolambkar et al., 2011a; Kolambkar et al., 2011b; Simmons et al., 2004).

Another key challenge in developing a biological implant for the treatment of degenerative joint diseases is engineering phenotypically stable cartilaginous tissues of sufficient scale to resurface the entire joint. This is particularly challenging in the context of OA, as only a limited number of therapeutically useful chondrocytes (CCs) can be isolated from diseased joints (Kock et al., 2012), and because CCs in OA cartilage produce cartilagedegrading enzymes, such as MMP13 and aggrecanases (Van der Kraan and Van den Berg, 2012). Furthermore, the expansion of CCs in vitro to obtain sufficient numbers of cells can lead to de-differentiation of cells towards a more fibroblast-like phenotype (Benya and Shaffer, 1982; Diaz-Romero et al., 2005). Mesenchymal stem cells (MSCs) can be used as an alternative to CCs for cartilage tissue-engineering (Mesallati et al., 2014b). MSCs possess the ability to proliferate extensively in vitro while maintaining their multipotent differentiation potential (Pittenger et al., 1999), making them an almost ideal cell type for engineering scaled-up cartilaginous constructs large enough to resurface an entire joint (Liu et al., 2014). However, as outlined previously, cartilage tissue-engineered using MSCs has been shown to become hypertrophic and undergo endochondral ossification in vivo (Farrell et al., 2011; Farrell et al., 2009; Janicki et al., 2010; Scotti et al., 2013; Scotti et al., 2010). Therefore, the second objective of this study was to explore two alternative strategies for engineering phenotypically stable cartilage tissue in vivo using stem cells. We first investigated if cartilage engineered in vitro using a scaffold-free or self-assembly (SA) approach, which has previously been shown to lead to the development of more hyaline cartilagelike tissue in vitro compared to hydrogel encapsulation (Mesallati et al., 2014a; Mesallati et al., 2014b), could lead to the development of more phenotypically stable cartilage in vivo. This was investigated using either BMSCs or infrapatellar fat pad-derived stem cells (FPSCs), as stem cells isolated from within the joint have been shown to have a gene expression profile more similar to chondrocytes than other chondroprogenitor cells, such as BMSCs (Segawa et al., 2009). Hence, the origin of stem cells (bone marrow or joint tissue) might influence the in vivo fate of cartilage tissues engineered using such cells. Control constructs were also implanted where the chondral layer of the osteochondral construct was engineered using stem cell laden agarose gels instead of the SA approach, as this hydrogel has previously been shown to promote hypoxia mediated chondrogenesis in vivo (Emans et al., 2010). Secondly, based on previous studies that demonstrate that co-culture of CCs and MSCs enhances cartilage matrix synthesis (Acharya et al., 2012; Tsuchiya et al., 2004; Wu et al., 2011) and suppresses markers of MSC hypertrophy (such as type $\mathrm{X}$ collagen expression) in vitro (Acharya et al., 2012; Bian et al., 2011; Fischer et al., 2010; Kang et al., 2012), we investigated if a co-culture of CCs and either BMSCs or FPSCs could be used to engineer a layer of phenotypically stable articular cartilage as part of an osteochondral construct in vivo.

The final objective of the study was to scale-up such an osteochondral construct in order to tissue-engineer an anatomically shaped biological implant that could potentially replace an entire diseased joint. It was hypothesised that this would be possible by combining scaled-up, anatomically shaped hypertrophic cartilaginous templates mimicking the geometry of the medial femorotibial joint (formed by injecting BMSC laden alginate into moulds fabricated by rapid prototyping) with engineered articular cartilage (formed by SA of co-cultured CCs and BMSCs). If this can be realised, it could lead to the development of a novel tissue-engineered therapy for the treatment of OA.

\section{Materials and Methods}

\section{Experimental design}

The first objective of the study was to determine the combination of cell and scaffold type that could be used to tissue-engineer phenotypically stable cartilage overlaying functional bone. Bilayered constructs were formed as shown in Fig. 1 (further details provided below). For all experimental groups, the bottom layer of these bilayered constructs, termed the endochondral or osseous layer, consisted of bone marrow derived mesenchymal stem cells (BMSCs) seeded within an alginate hydrogel. The top layer, termed the chondral layer, consisted of either an agarose hydrogel seeded with cells or a layer of 'selfassembled' or 'scaffold-free' tissue (formed from a high density layer of cells). To determine the effect of cell source on formation and development of cartilage within the top chondral layer of the osteochondral construct, these layers were formed using either chondrocytes (CCs) only, BMSCs only, infrapatellar fat pad-derived stem cells (FPSCs) only, a BMSC \& CC co-culture (4:1 ratio) or a FPSC \& CC co-culture (4:1 ratio) using either 


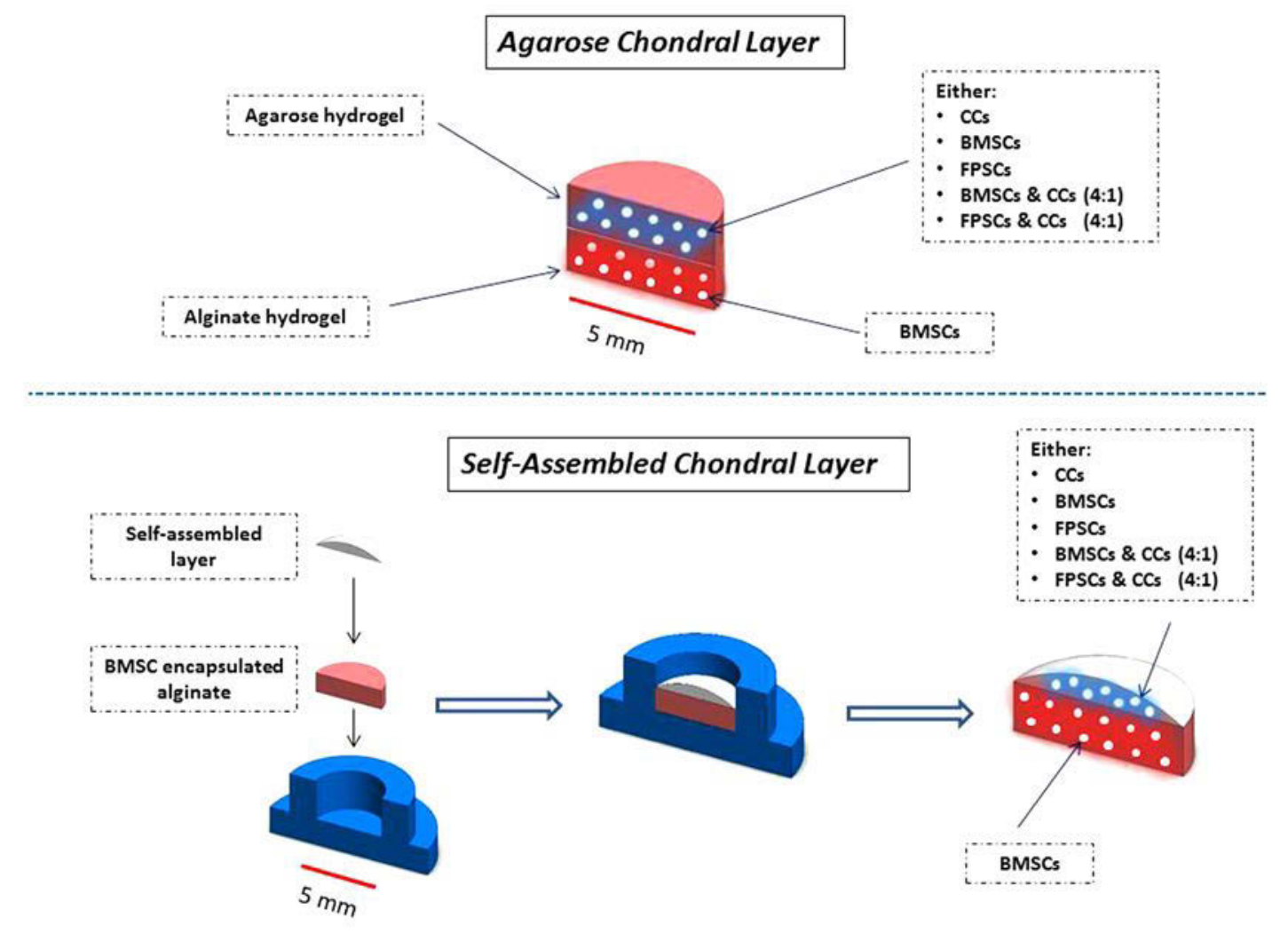

Fig. 1. Schematic of experimental design. The chondral layers, formed either through cell encapsulation into agarose hydrogels or using a self-assembly approach, were combined with BMSC laden alginate hydrogels (which formed the osseous or endochondral layer) in custom built moulds (blue moulds in figure) to form osteochondral constructs. CC, chondrocyte; BMSC, bone marrow-derived mesenchymal stem cell; FPSC, infrapatellar fat pad-derived stem cell.

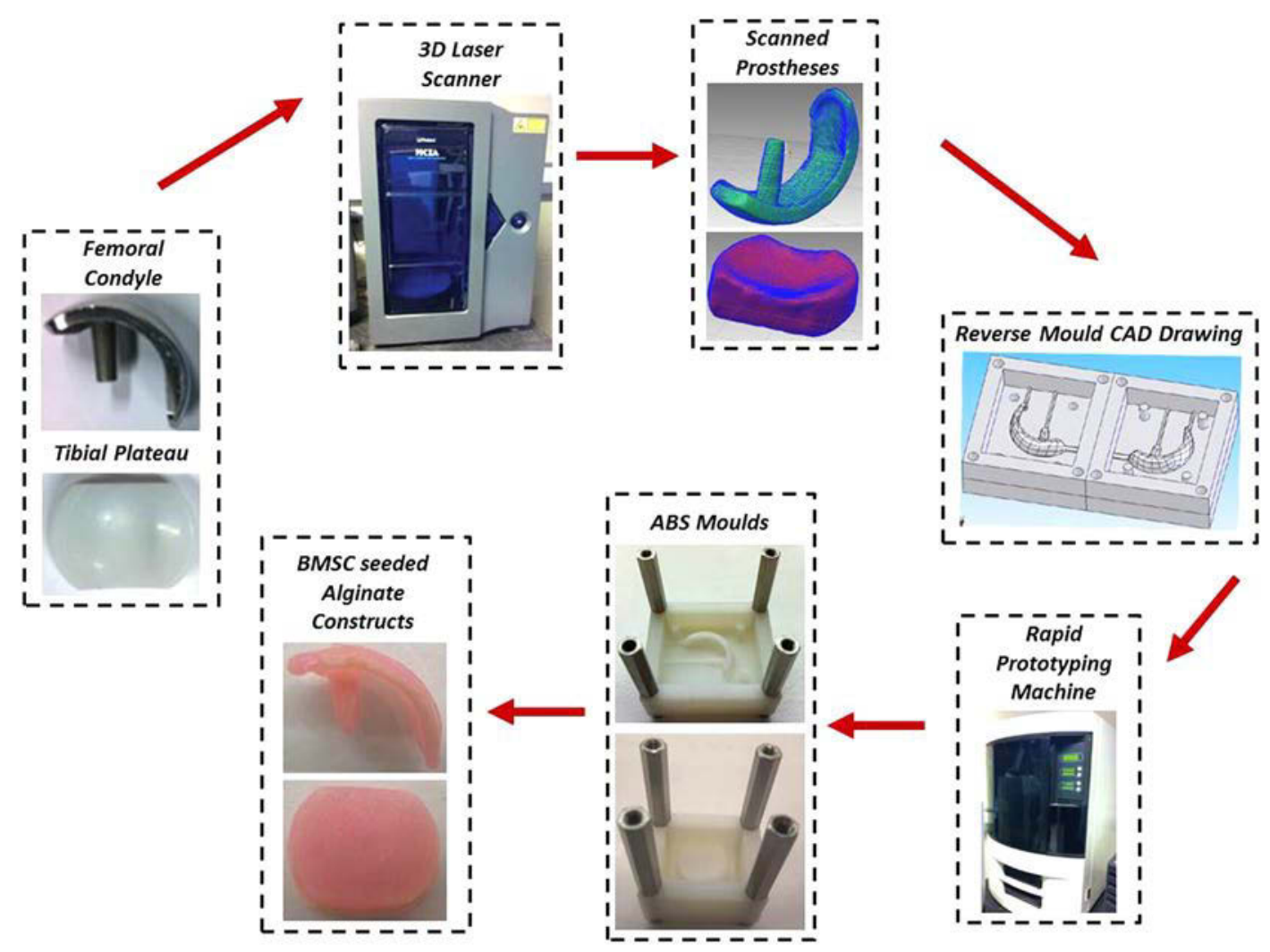

Fig. 2. Fabrication of scaled-up, anatomically shaped BMSC seeded alginate constructs in the shape of the femoral condyle and the tibial plateau. 
a self-assembly (SA) approach or agarose hydrogel encapsulation. Bi-layered constructs were maintained in vitro for 6 weeks in a chemically defined chondrogenic medium containing $10 \mathrm{ng} / \mathrm{mL}$ TGF- $\beta 3$. Constructs were subsequently implanted subcutaneously into the back of nude mice for a further 6 weeks.

In the second phase of the study, scaled-up BMSCseeded alginate constructs ( $\sim 2 \mathrm{~cm}$ diameter) mimicking the geometry of femorotibial joint replacement prostheses were generated from moulds fabricated using rapid prototyping (Fig. 2). Briefly, this involved firstly scanning unicondylar knee replacement prostheses in a 3D laser scanner. The scanned models were modified in SolidWorks to create moulds, and imported into a stratasys dimension fused deposition modelling (FDM) machine. This allowed for the creation of acrylonitrile butadiene styrene (ABS) moulds, from which large BMSC-seeded alginate constructs were fabricated. These scaled-up constructs were covered by a self-assembled layer ( $\sim 2 \mathrm{~cm}$ diameter $)$ of engineered cartilaginous tissue (formed through BMSC \& CC co-culture). After 6 weeks of in vitro culture in chondrogenic media, the scaled-up constructs were implanted subcutaneously into nude mice for a further 8 weeks.

\section{Cell isolation and expansion}

Articular cartilage was aseptically harvested from porcine femoral condyles (4 months old) and the cartilage slices were rinsed thoroughly with Dulbecco's phosphate buffered saline (Sigma-Aldrich, Dublin, Ireland; PBS) containing penicillin $(200 \mathrm{U} / \mathrm{mL})$-streptomycin $(100 \mu \mathrm{g} / \mathrm{mL})$ (GIBCO, Invitrogen, Dublin, Ireland), and amphotericin B $(2.5 \mu \mathrm{g} / \mathrm{mL})$ (Sigma-Aldrich) (Mesallati et al., 2014a). Chondrocytes (CCs) were isolated from cartilage slices overnight by digestion with high-glucose Dulbecco's modified Eagle's medium GlutaMAX $(4.5 \mathrm{mg} / \mathrm{mL}$ D-Glucose, 200 mM L-Glutamine; hgDMEM) (GIBCO, Invitrogen) containing collagenase type II $(350 \mathrm{U} / \mathrm{mL})$ (Worthington, Langanbach Services, Bray, Ireland) for $14 \mathrm{~h}$ under constant rotation at $37^{\circ} \mathrm{C}$. The resulting cell suspension was passed through a $40 \mu \mathrm{m}$ pore-size cell sieve (Fisher Scientific, Dublin, Ireland) and the filtrate centrifuged and rinsed with PBS twice. Cell number and viability were determined using a haemocytometer and $0.4 \%$ trypan blue staining (Sigma-Aldrich).

Infrapatellar fat pads (IFPs) were harvested from 4 month old porcine femoropatellar joints and diced followed by 3-4 h incubation with hgDMEM GlutaMAX containing collagenase type II (750 U/mL) (Buckley and Kelly, 2012). After tissue digestion, the resulting cell suspension was passed through a $40 \mu \mathrm{m}$ pore-size cell sieve and the filtrate centrifuged and rinsed twice with PBS. Bone marrowderived MSCs (BMSCs) were isolated from the femora of one porcine donor (4 months old). BMSCs were isolated and expanded based on a modified protocol developed for human MSCs (Lennon and Caplan, 2006).

CCs, infrapatellar fat pad-derived stem cells (FPSCs) and BMSCs were plated at a seeding density of $5 \times 10^{3}$ cells $/ \mathrm{cm}^{2}$ in $500 \mathrm{~cm}^{2}$ triple flasks (Thermo Fisher Scientific, Dublin, Ireland) and expanded to passage two (P2) in a humidified atmosphere at $37{ }^{\circ} \mathrm{C}$ and $5 \% \mathrm{CO}_{2}$. All cells were maintained in hgDMEM GlutaMAX supplemented with $10 \% \mathrm{v} / \mathrm{v}$ foetal bovine serum (GIBCO, Invitrogen; FBS $)$, penicillin $(100 \mathrm{U} / \mathrm{mL})$-streptomycin $(100 \mu \mathrm{g} / \mathrm{mL})$ and $5 \mathrm{ng} / \mathrm{mL}$ human fibroblast growth factor-2 (ProSpecTany TechnoGene Ltd., Israel; FGF-2) during all expansion phases.

\section{Formation of the osseous and chondral layer of the bi-layered constructs}

The first objective of the study was to determine the combination of cell and scaffold type that could be used to tissue-engineer phenotypically stable cartilage overlaying functional bone in vivo (Fig. 1). The in vitro development of these bi-layered constructs consisted of two phases. In the first phase, each individual layer was cultured separately for 2 weeks. In the second phase, the two layers were then stacked and maintained in culture for a further 4 weeks prior to implantation. The osseous (bottom) layers of all bilayered/osteochondral constructs consisted of a cylindrical alginate hydrogel seeded with BMSCs. To create these cylindrical alginate constructs, an agarose/ calcium chloride solution was first created by mixing $6 \%$ molten agarose (routine agarose; Sigma Aldrich) with $100 \mathrm{mM} \mathrm{CaCl}_{2}$ (Sigma-Aldrich) in a 1:1 ratio. This solution was poured into a custom-built Tufset polyurethane mould to create an agarose $/ \mathrm{CaCl}_{2}$ mould consisting of multiple wells of diameter $5 \mathrm{~mm}$ and thickness $2 \mathrm{~mm}$. The final concentration of this mould was $3 \%$ agarose $/ 50 \mathrm{mM}$ $\mathrm{CaCl}_{2}$. Next, $2 \% \mathrm{w} / \mathrm{v}$ alginate (Pronova UP LVG, FMC BioPolymer, Norway) was dissolved overnight in PBS, sterile filtered and encapsulated with P2 BMSCs (20 million cells per $\mathrm{mL}$ ). The cell encapsulated alginate solution was pipetted into the wells of the agarose $/ \mathrm{CaCl}_{2}$ mould, and allowed to cross-link ionically with the $\mathrm{CaCl}_{2}$ contained within the mould for $30 \mathrm{~min}$. After this time, stable and solid BMSC seeded alginate hydrogels ( $5 \mathrm{~mm}$ diameter, $2 \mathrm{~mm}$ thick; 800,000 cells per construct) were removed from the wells and washed twice with basic chemically defined medium (basic CDM). Basic CDM consisted of hgDMEM GlutaMAX supplemented with penicillin $(100 \mathrm{U} / \mathrm{mL})$-streptomycin $(100 \mu \mathrm{g} / \mathrm{mL})$, $100 \mu \mathrm{g} / \mathrm{mL}$ sodium pyruvate, $40 \mu \mathrm{g} / \mathrm{mL}$ L-proline, and $1.5 \mathrm{mg} / \mathrm{mL}$ bovine serum albumin (all Sigma-Aldrich). BMSC seeded alginate constructs were then cultured for 2 weeks in defined CDM, consisting of basic CDM supplemented with $0.25 \mu \mathrm{g} / \mathrm{mL}$ amphotericin $\mathrm{B}, 1 \times$ insulintransferrin-selenium, $4.7 \mu \mathrm{g} / \mathrm{mL}$ linoleic acid, $50 \mu \mathrm{g} / \mathrm{mL}$ L-ascorbic acid-2-phosphate, $100 \mathrm{nM}$ dexamethasone (all Sigma-Aldrich), and $10 \mathrm{ng} / \mathrm{mL}$ TGF- $\beta 3$ (ProSpec-Tany TechnoGene Ltd., Israel).

The top layer of the bilayered constructs (termed the chondral layer) was formed next. This layer consisted of either a cell seeded agarose hydrogel or a layer of scaffoldfree cartilage generated using SA of cells. Cylindrical agarose hydrogels ( $5 \mathrm{~mm}$ diameter, $1.5 \mathrm{~mm}$ thickness) were formed by mixing cell suspensions in basic CDM with $4 \%$ agarose in sterile PBS (Mesallati et al., 2013). Solutions were mixed at a ratio of $1: 1$ at $\sim 40^{\circ} \mathrm{C}$, to yield a final gel concentration of $2 \%$ and a cell seeding density 
of 20 million cells $/ \mathrm{mL}$ (600,000 cells per construct). The agarose/cell suspensions were cast in a stainless steel mould, allowed to cool for $30 \mathrm{~min}$, and solid construct cylinders were removed using a biopsy punch. Such agarose constructs were formed using chondrocytes (CCs) alone, BMSCs alone, infrapatellar fat pad-derived stem cells (FPSCs) alone, a BMSC \& CC co-culture (4:1 ratio) and a FPSC \& CC co-culture (4:1 ratio). All cells had been expanded to $\mathrm{P} 2$. These constructs were cultured for 2 weeks in defined CDM.

Self-assembled constructs were formed as previously described (Mesallati et al., 2014b). Briefly, 4 million cells in $250 \mu \mathrm{L}$ aliquots of expansion medium (hgDMEM GlutaMAX supplemented with $10 \% \mathrm{v} / \mathrm{v}$ FBS and penicillin-streptomycin) were added to $6.5 \mathrm{~mm}$ diameter Transwell cell culture inserts (Corning Transwell ${ }^{\circledR}$, VWR, Dublin, Ireland), seated in 6 well plates (Fisher Scientific, Dublin, Ireland). These cell seeding numbers equated to approximately $1.2 \times 10^{5}$ cells $/ \mathrm{mm}^{2}$ of Transwell membrane. Each Transwell insert consisted of a polyethylene terephthalate (PET) membrane containing $3 \mu \mathrm{m}$ pores. Cells were maintained in expansion medium for $24 \mathrm{~h}$, after which medium was switched to defined CDM for a further 2 weeks of culture. Self-assembled constructs were formed using CCs alone, BMSCs alone, FPSCs alone, a BMSC $\&$ CC co-culture (4:1 ratio) and a FPSC \& CC co-culture (4:1 ratio). All cells had been expanded to $\mathrm{P} 2$.

\section{In vitro development of osteochondral constructs}

After 2 weeks in defined CDM, self-assembled constructs were carefully removed from their transwells; $5 \mathrm{~mm}$ diameter samples were then cored from the $6.5 \mathrm{~mm}$ diameter Transwell self-assembled constructs (each new $5 \mathrm{~mm}$ construct was equivalent to $\sim 2.4$ million cells). Agarose and alginate gels were also removed from isolated culture at this point. Bilayered constructs were then formed by first confining the osseous layer (BMSC seeded alginate gels cultured as described above) within $5 \mathrm{~mm}$ diameter custom made agarose moulds (Fig. 1), and placing the chondral layer (either $5 \mathrm{~mm}$ diameter agarose gels or self-assembled constructs cultured as described above) on top of the osseous layer. These confined osteochondral constructs were cultured in defined CDM for 2 weeks in vitro, before being removed from confinement and cultured for 2 additional weeks (total of 6 weeks in vitro culture). To prevent alginate gels from degrading in the culture medium (due to release of divalent ions from the gels), the chondrogenic medium was supplemented with $0.25 \mathrm{mM}$ $\mathrm{CaCl}_{2}$ for the final 2 weeks of the in vitro culture period. Constructs were subsequently implanted subcutaneously into the back of nude mice.

\section{Subcutaneous implantation in nude mice}

Following 6 weeks in vitro priming, $5 \mathrm{~mm}$ diameter bilayered constructs ( $n=9$ per group) were implanted subcutaneously into the back of nude mice (Balb/c; Harlan, UK). This involved creating two subcutaneous pockets along the central line of the spine (at shoulders and hips), and inserting three constructs into each pocket. Nine constructs were implanted per experimental group.
Mice were sacrificed 6 weeks post-implantation, by $\mathrm{CO}_{2}$ inhalation. The animal protocol was reviewed and approved by the ethics committee of Trinity College Dublin.

\section{Generating scaled-up anatomically shaped osteochondral constructs}

In order to generate scaled-up tissue-engineered osteochondral constructs, mimicking the geometry of knee implants (Fig. 2), scans were taken of prostheses of both the femoral condyle and tibial plateau using a PICZA 3D laser scanner (model LPX-250). The program Pixform was used to render and mesh the scans taken from the laser scanner and subsequently assemble 3D solid models of the constructs. Next, these model files were imported into SolidWorks. The femoral condyle construct was sectioned in half to design a two-part reverse mould drawing of the original, while the tibial plateau construct was used to design a single inverse mould. A rapid prototyping machine (Stratasys dimension FDM) was then used to create acrylonitrile butadiene styrene (ABS) moulds of the SolidWorks designs through fused deposition modelling.

A solution of $8 \%$ molten agarose (routine agarose) mixed with $100 \mathrm{mM} \mathrm{CaCl}_{2}$ (1:1 ratio) was then used to fill sterilised ABS moulds, thus creating a two-part agarose/ $\mathrm{CaCl}_{2}$ mould for the femoral condyle, and a single agarose/ $\mathrm{CaCl}_{2}$ mould for the tibial plateau (final concentration of $4 \%$ agarose and $50 \mathrm{mM} \mathrm{CaCl}_{2}$ ). For the femoral condyle, the two-part agarose $/ \mathrm{CaCl}_{2}$ moulds were fitted together and filled with a mixture of BMSCs (P2) and uncrosslinked alginate $(2 \% \mathrm{w} / \mathrm{v})$. By using these moulds, $\mathrm{CaCl}_{2}$ was allowed to diffuse through the agarose and crosslink the alginate to form scaled-up, anatomically shaped constructs in the shape of the femoral condyle $(2 \mathrm{~cm}$ diameter). The alginate was injected using a hypodermic needle and syringe, through an infiltration port included in the original mould design. A similar process was used to form scaled-up BMSC seeded alginate constructs in the shape of the tibial plateau (dimensions $18 \mathrm{~mm} \times 12 \mathrm{~mm} \times 6 \mathrm{~mm}$ ). These alginate constructs were seeded at 20 million cells/ $\mathrm{mL}$ (equating to $\sim 15$ million cells per femoral construct, and $\sim 20$ million cells per tibial construct). These scaled-up alginate constructs, which ultimately formed the osseous region of scaled-up osteochondral constructs, were cultured for 2 weeks in defined CDM supplemented with $1 \mathrm{mM}$ $\mathrm{CaCl}_{2}$.

The articular or chondral layers of the scaled-up osteochondral constructs were formed using a scaffoldfree or SA approach similar to that described previously. BMSCs and CCs were expanded to P2, trypsinised, and mixed together to form a homogenous cell suspension (BMSC to CC ratio of 4:1). This cell suspension was then used to form self-assembled constructs on PET Transwell membranes confined within custom made polydimethylsiloxane (PDMS) moulds. Self-assembled layers for the femoral condyle were $20 \mathrm{~mm} \times 6 \mathrm{~mm}$, whilst those for the tibial plateau were $18 \mathrm{~mm} \times 12 \mathrm{~mm}$; they were formed using the same cell $/ \mathrm{mm}^{2}$ value as used in the first SA phase of the study $\left(1.2 \times 10^{5}\right.$ cells $\left./ \mathrm{mm}^{2}\right)$. This equated to $\sim 15$ million cells per femoral SA layer, and $\sim 25$ million cells per tibial SA layer. SA constructs were cultured for 2 
weeks in defined CDM. After this time, they were combined with their corresponding scaled-up BMSC-alginate bases described above to create scaled-up, anatomically shaped osteochondral constructs. The osseous region and selfassembled chondral layer were initially attached using a small amount of fibrin gel (to act like glue). Osteochondral constructs were cultured for 4 additional weeks in vitro in defined CDM supplemented with $1 \mathrm{mM} \mathrm{CaCl}_{2}$ (6 weeks total culture pre-implantation). In addition, the engineered tibial implant was implanted subcutaneously into the back of nude mice (Balb/c, Harlan). A subcutaneous pocket was created to the side of the central line of the spine, and one construct was implanted per animal $(n=2)$. Mice were sacrificed 8 weeks post-implantation.

\section{Biochemical analysis}

The biochemical content of $5 \mathrm{~mm}$ diameter osteochondral constructs $(n=5)$ was assessed at the beginning of the experiment (day 0), pre-implantation (day 42) and postimplantation (day 84). Prior to biochemical analysis, osteochondral constructs were sliced at the interface to separate the top chondral layer and bottom osseous layer. These separated layers were then sliced in half, washed in DI water, weighed and frozen for subsequent analysis. The first half of each layer was digested in papain $(125 \mu \mathrm{g} /$ $\mathrm{mL}$ ) in $0.1 \mathrm{M}$ sodium acetate, $5 \mathrm{mM}$ L-cysteine-HCL, 0.05 M EDTA, pH 6 (all Sigma-Aldrich) under constant rotation at $60{ }^{\circ} \mathrm{C}$ for $18 \mathrm{~h}$. After this time samples were rotated in $55 \mathrm{mM}$ sodium citrate at $37{ }^{\circ} \mathrm{C}$ for $40 \mathrm{~min}$ to allow all alginate to dissolve. DNA content of constructs was quantified using the Hoechst Bisbenzimide 33258 dye assay (Kim et al., 1988). Proteoglycan content was estimated by quantifying the amount of sulphated glycosaminoglycans (sGAG) in each construct using the dimethylmethylene blue (DMMB) dye binding assay (Blyscan, Biocolor Ltd., Carrickfergus, UK), with a shark chondroitin sulphate standard. The $\mathrm{pH}$ of the DMMB dye was adjusted to 1.5 to limit detection of carboxylated alginate during the sGAG assay (Enobakhare et al., 1996). Total collagen content of constructs was determined by measuring the hydroxyproline content using the dimethylaminobenzaldehyde and chloramine $\mathrm{T}$ assay (Kafienah and Sims, 2004), using a hydroxyproline to collagen ratio of 1:7.69 (Ignat'eva et al., 2007). The second half of each layer was digested in $1 \mathrm{M}$ hydrochloric acid at $60{ }^{\circ} \mathrm{C}$ and $10 \mathrm{rpm}$ for $18 \mathrm{~h}$. The calcium content was then determined using a Sentinel Calcium kit (Alpha Laboratories Ltd., UK). Scaled-up anatomically shaped osteochondral constructs were assessed pre- and postimplantation, using the same techniques as described above.

\section{Histology and immunohistochemistry}

At the end of each experiment, at least 2 samples per experimental group were fixed overnight in $4 \%$ paraformaldehyde (Sigma-Aldrich) supplemented with barium chloride (in order to permanently crosslink the alginate matrix and constructs). Post-implantation osteochondral constructs ( $5 \mathrm{~mm}$ diameter) were decalcified in EDTA for 7 days prior to wax embedding. Likewise, scaled-up anatomically shaped constructs were decalcified for $14 \mathrm{~d}$. Samples were dehydrated with a graded series of alcohol and embedded in paraffin. $10 \mu \mathrm{m}$ sections were produced of the cross section perpendicular to the construct face. Sections were first stained with haematoxylin and eosin (H\&E) and $1 \%$ alcian blue 8GX (Sigma-Aldrich) in $0.1 \mathrm{M}$ HCL for sGAG accumulation, with a counter stain of nuclear fast red to assess cellular distribution. Sections were next stained with alcian blue and aldehyde fuchsin to differentiate between cartilage sGAG deposition and residual alginate. The final stains involved $1 \%$ alizarin red to assess mineral accumulation and picro-sirius red (Sigma-Aldrich) to visualise collagen accumulation.

Collagen types I, II and X deposition were identified by immunohistochemical analysis. Briefly, sections were treated with peroxidase, followed by treatment with chondroitinase ABC (Sigma-Aldrich) in a humidified environment at $37{ }^{\circ} \mathrm{C}$ to enhance permeability of the extracellular matrix. Sections were then incubated with goat serum to block non-specific sites, before the primary antibody was applied to the sections. Collagen type I (ab90395, 1:400, $1 \mathrm{mg} / \mathrm{mL}$ ), collagen type II (ab3092, $1: 100,1 \mathrm{mg} / \mathrm{mL})$ or collagen type X (ab49945, 1:100, $1.4 \mathrm{mg} / \mathrm{mL}$ ) primary antibodies (mouse monoclonal, Abcam, Cambridge, UK) were applied for $1 \mathrm{~h}$ at room temperature. Next, the secondary antibody (for collagen types I and II, Anti-Mouse IgG biotin conjugate, 1:200, $2.1 \mathrm{mg} / \mathrm{mL}$, Sigma-Aldrich; for collagen type X, ab49760 Anti-Mouse IgM mu chain (Biotin), 1:100, $0.1 \mathrm{mg} /$ $\mathrm{mL}$, Abcam) was added for $1 \mathrm{~h}$ followed by incubation with ABC reagent (Vectastain PK-400, Vector Labs, Peterborough, UK) for $45 \mathrm{~min}$. Finally, sections were developed with DAB peroxidase (Vector Labs) for $5 \mathrm{~min}$. Positive and negative controls were included in the immunohistochemistry staining protocol for each batch.

\section{Micro-computed tomography}

Micro-computed tomography $(\mu \mathrm{CT})$ scans were performed using a Scanco Medical $40 \mu \mathrm{CT}$ system (Scanco Medical, Bassersdorf, Switzerland) in order to quantify mineral content and to assess mineral distribution in all osteochondral constructs post implantation. For all $5 \mathrm{~mm}$ diameter osteochondral constructs, samples were scanned at the end of the 6 week in vivo time period. Constructs were scanned in DI water, at a voxel resolution of $16 \mu \mathrm{m}$, a voltage of $70 \mathrm{kVp}$, and a current of $114 \mu \mathrm{A} .3$ constructs were analysed per experimental group. For scaled-up, anatomically shaped osteochondral constructs, samples were scanned at the end of the 8 week in vivo time period.

\section{Statistical analysis}

Statistical analyses were performed using the software package MINITAB 15.1 (Minitab Ltd., Coventry, UK). Groups were analysed for significant differences using a general linear model for analysis of variance. Tukey's test for multiple comparisons was used to compare conditions. A Box-Cox transformation was used to normalise data sets where necessary. Significance was accepted at a level of $p \leq 0.05$. Numerical and graphical results are presented as mean \pm standard deviation $(n=5)$, with graphical results produced using GraphPad Prism (Version 6.02). 
a

Experimental Set-up

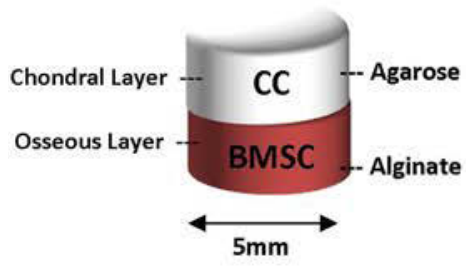

Chondral -

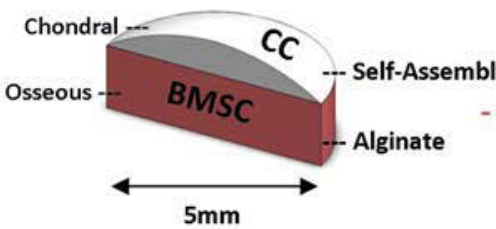

$5 \mathrm{~mm}$

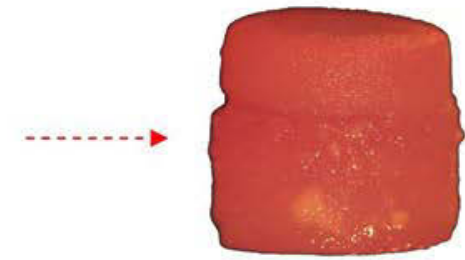

\section{b}

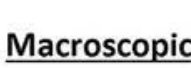

$1 \mathrm{~mm}$

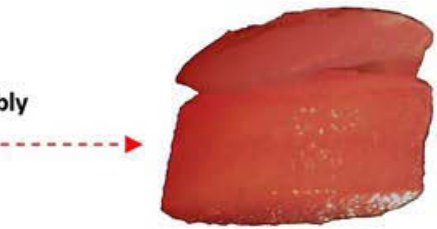

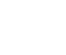

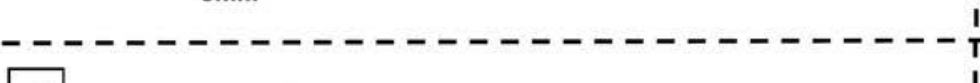

d
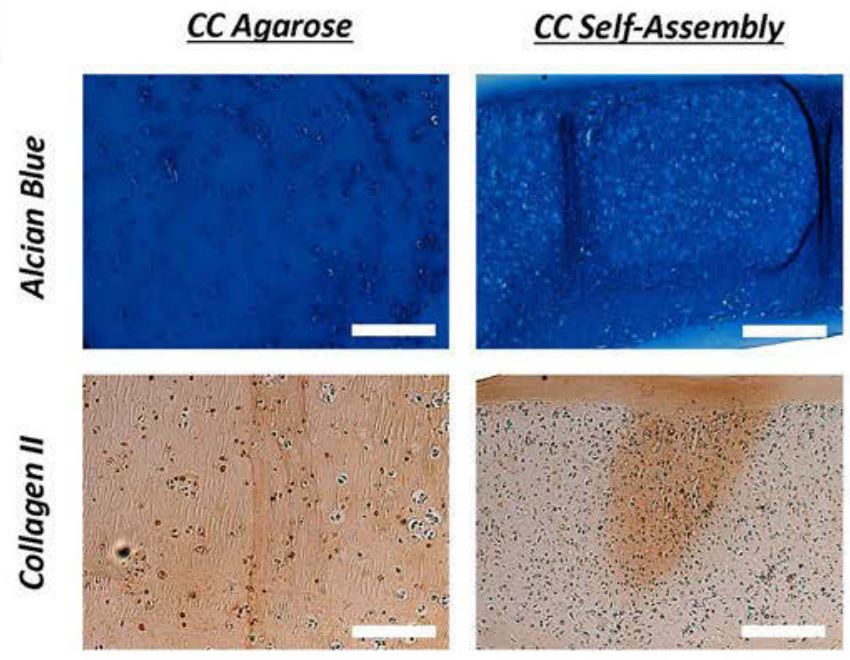

\section{Chondral \\ Layer}

c
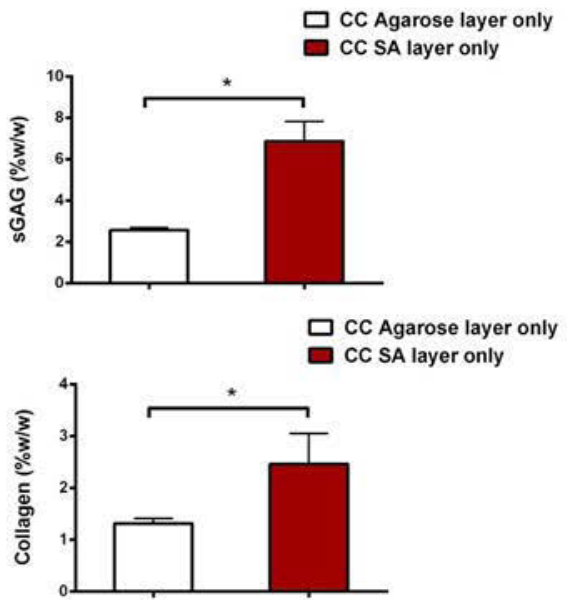

e BMSC Alginate
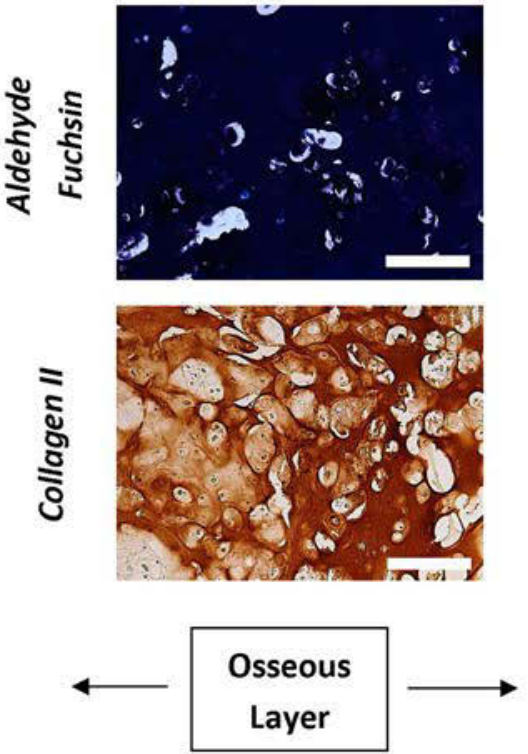

Fig. 3. Bilayered constructs pre-implantation, formed using chondrocytes (CCs) in the chondral layer and bone marrowderived MSCs (BMSCs) in the bottom osseous alginate layer. (a) Schematic of bilayered constructs, with chondral layers formed through agarose encapsulation or self-assembly (SA). (b) Macroscopic images after 6 weeks in vitro culture. (c) sGAG and collagen accumulation $(\% \mathrm{w} / \mathrm{w})$ within the chondral layer of these osteochondral constructs. (d) Alcian blue staining for sGAG production and type II collagen immunohistochemistry of chondral layers. (e) Histological staining of osseous alginate layers. ${ }^{*}$ denotes significant difference with $p<0.05$. Scale bar $=200 \mu \mathrm{m}$.

\section{Results}

The osseous region of an osteochondral construct can be engineered using a chondrogenically primed BMSC laden alginate hydrogel that undergoes endochondral ossification in vivo

We have previously shown that it is possible to engineer stable cartilaginous tissue on top of a layer of mineralised hypertrophic cartilage by seeding chondrocytes (CCs) into the top 'chondral' layer of bilayered agarose hydrogels, and BMSCs into the bottom 'osseous' layer of these bilayered agarose hydrogels (Sheehy et al., 2013). The main limitation to this method was that the agarose did not degrade in vivo and hence prevented vascularisation and any subsequent endochondral ossification of the mineralised cartilaginous tissue in the bottom 'osseous' layer from occurring. As an alternative biomaterial, alginate hydrogels were seeded with BMSCs in this study and used to form the 'osseous' or 'endochondral' layer of bilayered constructs, where the overlaying articular cartilage or 'chondral' layer was formed using either an agarose hydrogel seeded with chondrocytes (CCs) or a layer of scaffold-free cartilage engineered through self-assembly (SA) of CCs (Fig. 3a, b). After 6 weeks in chondrogenic 


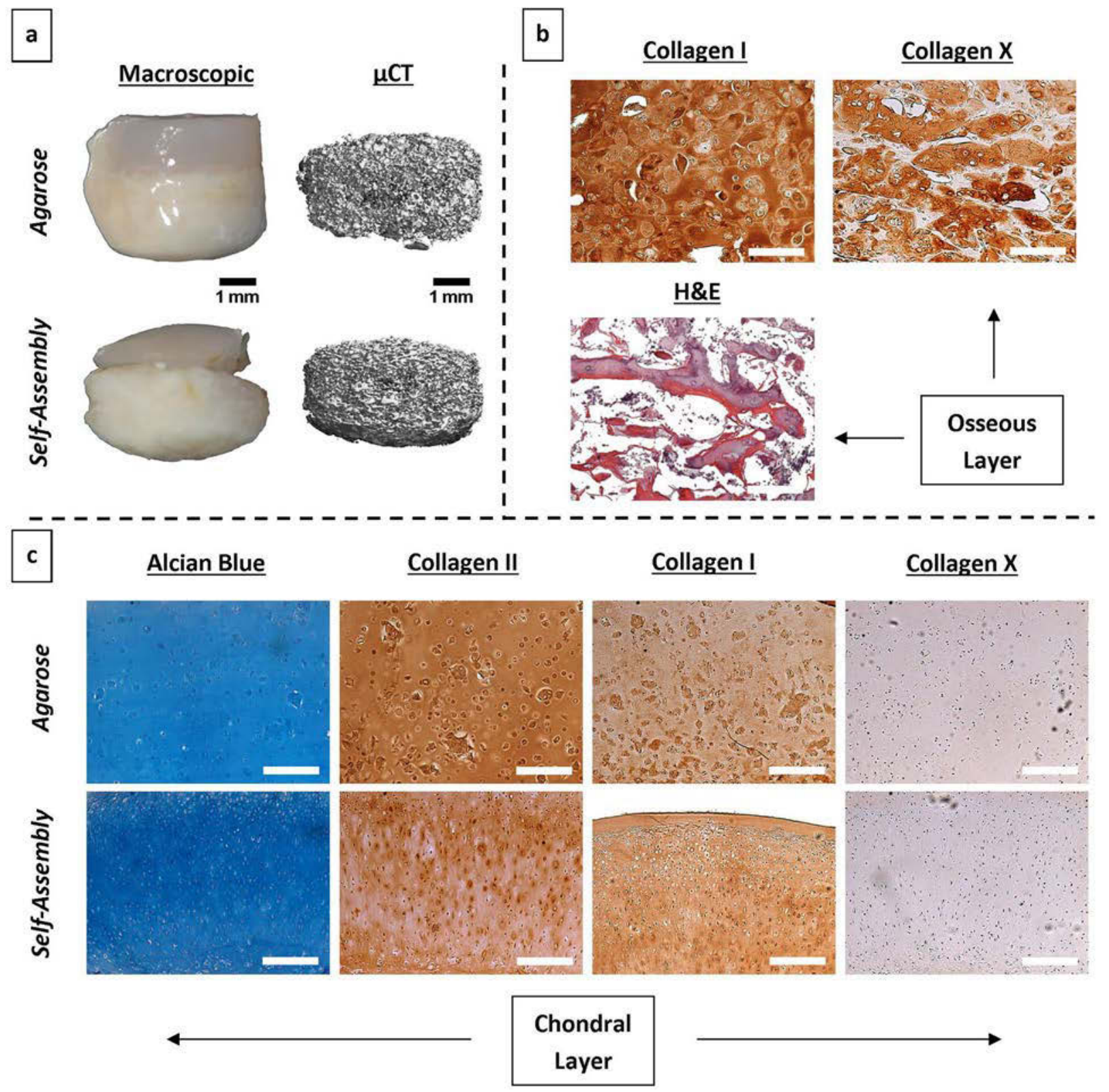

Fig. 4. Osteochondral constructs post-implantation, with chondral layers formed using only chondrocytes (CCs). (a) Macroscopic images and $\mu \mathrm{CT}$ scans of bilayered constructs, with chondral layers formed through agarose or self-assembly. (b) Collagen types I and X immunohistochemistry and H\&E staining of osseous alginate layers of osteochondral constructs (representative of all alginate samples). (c) Alcian blue staining and collagen immunohistochemistry of chondral layers. Scale bar $=200 \mu \mathrm{m}$.

media the SA approach generated a cartilage layer with significantly higher levels of sGAG and collagen (as a \% of tissue wet weight) compared to agarose encapsulation (Fig. 3c) (SA constructs reached levels of $6.9 \pm 1.0 \% \mathrm{w} / \mathrm{w}$ sGAG, $2.5 \pm 0.6 \% \mathrm{w} / \mathrm{w}$ collagen; agarose constructs reached levels of $2.6 \pm 0.1 \% \mathrm{w} / \mathrm{w}$ sGAG, $1.3 \pm 0.1 \% \mathrm{w} / \mathrm{w}$ collagen). Both approaches generated cartilaginous tissue staining positively for sGAG (alcian blue staining) and type II collagen (Fig. 3d). The underlying BMSC laden alginate layer also stained positively for SGAG (aldehyde fuchsin staining) and type II collagen (Fig. 3e) prior to implantation.

Following subcutaneous implantation into nude mice, the osseous region (BMSC laden alginate hydrogels) of the bi-layered constructs appeared hard and calcified (Fig. 4a). $\mu \mathrm{CT}$ analysis demonstrated extensive mineralisation in the alginate layers (Fig. 4a). The calcium content of the underlying osseous region of the osteochondral constructs was not affected by the approach (either agarose encapsulation or SA) used to engineer the overlaying chondral layer (data not shown). H\&E staining of the osseous region of the constructs revealed the formation of bony-like tissue (Fig. 4b). Collagen types I and X were also detected in this osseous layer (Fig. 4b), suggesting the formation of bone through the process of endochondral ossification. These results demonstrate that endochondral ossification can progress in the osseous region of the implant, despite the presence of chondrocytes in the 


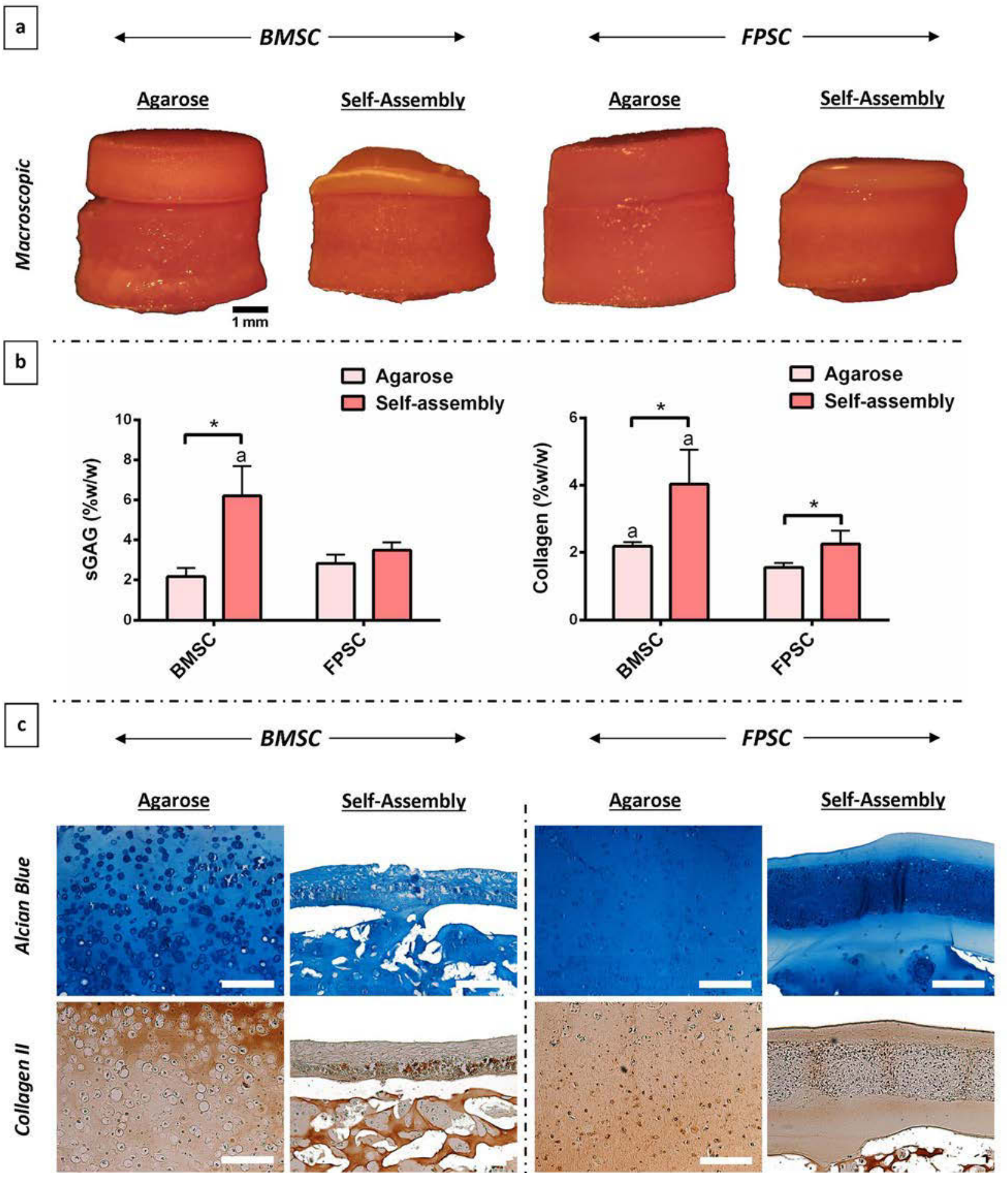

Fig. 5. Osteochondral constructs pre-implantation, with chondral layers formed using either bone marrow-derived MSCs (BMSCs) or infrapatellar fat pad-derived stem cells (FPSCs). (a) Macroscopic images of bilayered constructs after 6 weeks in vitro culture, with chondral layers formed through agarose or self-assembly. (b) sGAG and collagen accumulation $(\% \mathrm{w} / \mathrm{w})$ within the chondral layers of these osteochondral constructs. (c) Alcian blue staining and type II collagen immunohistochemistry of chondral layers. ${ }^{\mathrm{a}} p<0.05$ versus corresponding FPSC group in same scaffold. $*$ denotes significant difference with $p<0.05$. Scale bar $=200 \mu \mathrm{m}$.

top chondral region of the implant. A phenotypically stable cartilage tissue was generated in this top chondral layer of the osteochondral construct in vivo (Fig. 4c), with positive staining for sGAG and type II collagen, irrespective of whether this tissue was engineered using agarose encapsulation or SA. There was some staining of collagen type I in the cartilage generated by these expanded chondrocytes, with no evidence of collagen type $\mathrm{X}$ deposition observed (Fig. 4c). No mineralisation was detected in the chondral layers using $\mu \mathrm{CT}$ analysis (Fig. $4 a)$. 
Self-assembled cartilaginous grafts engineered using either bone marrow or infrapatellar fat pad-derived stem cells fail to form phenotypically stable tissue in vivo

While a phenotypically stable layer of articular cartilage can be engineered on an osteochondral construct using primary CCs alone, from a clinical perspective it may not be feasible to obtain the large number of CCs required to engineer a graft capable of resurfacing an entire joint. Given that stem cell derived cartilage grafts engineered using a scaffold-free or SA approach have been shown to lead to the generation of higher quality articular cartilage-like tissues in vitro compared to agarose hydrogel encapsulation (Mesallati et al., 2014b), we next sought to determine if this would translate into the development of a more phenotypically stable tissue in vivo. To this end, the previous study was repeated, using stem cells isolated from different tissues in place of CCs to engineer the chondral layer of the osteochondral constructs, again using either agarose encapsulation or a SA approach (Fig. 5a).

We found that both BMSCs and FPSCs could be used (using either agarose encapsulation or SA) to engineer cartilaginous tissues in vitro. The highest levels of sGAG and collagen accumulation $(\% \mathrm{w} / \mathrm{w})$ were observed in the chondral layers generated using SA with BMSCs $(6.2 \pm 1.5 \% \mathrm{w} / \mathrm{w}$ sGAG; $4 \pm 1 \% \mathrm{w} / \mathrm{w}$ collagen) (Fig. $5 \mathrm{~b}$ ). Both BMSCs and FPSCs (using either agarose encapsulation or SA) generated chondral layers rich in proteoglycans and type II collagen (Fig. 5c).

Post-implantation, both the chondral and osseous layers of constructs engineered using BMSCs appeared hard and calcified (Fig. 6a). The chondral layers of osteochondral constructs generated using FPSCs were macroscopically different, with a more fibrous or fibrocartilaginous appearance (Fig. 6a). $\mu \mathrm{CT}$ analysis demonstrated that mineralisation occurred in the osseous (BMSC seeded alginate) layer of all bilayered constructs, and furthermore, that the chondral layer of constructs engineered using BMSCs (agarose and SA) also mineralised (Fig. 6a). The chondral layer of constructs engineered using BMSCs accumulated the highest levels of calcium (highest levels of $11.4 \pm 1.3 \% / \mathrm{w} / \mathrm{w}$ in BMSC SA), with significantly lower levels of calcium observed in the FPSC seeded chondral layers (Fig. 6c).

Post-implantation, the chondral layers of all constructs engineered, using BMSCs or FPSCs, stained positively for sGAG and collagen type II accumulation. However, staining was generally weaker than that found in the chondral layers generated using CCs (Fig. 6b), with particularly weak staining for sGAG. Conversely, staining of collagen types I and X was much stronger in the chondral layers engineered using stem cells, compared to respective CC chondral layers (for both agarose and SA) (Fig. 6b). In addition, chondral layers engineered using SA of either BMSCs or FPSCs were considerably thinner than those generated using CCs.
A co-culture of chondrocytes (CCs) and either BMSCs or FPSCs enhances the in vitro development of engineered cartilage and in vivo leads to the development of a more phenotypically stable tissue We next sought to determine if phenotypically stable cartilage could be generated in vivo using a co-culture of CCs and either BMSCs or FPSCs. The chondral layers of bilayered constructs were created by SA of co-cultured stem cells and CCs in a 4:1 ratio (Fig. 7a). Self-assembled cartilaginous tissues, engineered using such a co-culture of CCs and either BMSCs or FPSCs, were found to be considerably thicker pre-implantation than their respective stem cell only controls (Fig. 7a). For BMSC \& CC cocultures, these tissues also stained more intensely in vitro for sGAG and type II collagen than BMSC only controls. A cartilage tissue-engineered using a co-culture of CCs and either BMSCs or FPSCs contained significantly higher levels of both sGAG and collagen pre-implantation, compared to those engineered using either stem cell type alone (Fig. 7b). No significant differences were observed in self-assembled constructs when matrix levels were normalised to tissue wet weight, due to the variance in weight of the relatively light SA layers (sGAG $(\% \mathrm{w} / \mathrm{w})$ : CC only $-6.9 \pm 0.9$; BMSC only $-6.2 \pm 1.5$; BMSC $\&$ CC $-4.5 \pm 0.6$; FPSC only $-3.5 \pm 0.4$; FPSC \& CC $-5.1 \pm 1.1$ and collagen $(\% \mathrm{w} / \mathrm{w})$ : $\mathrm{CC}$ only $-2.5 \pm 0.6$; BMSC only $-4 \pm 1$; BMSC \& CC $-2.7 \pm 0.5$; FPSC only $-2.3 \pm 0.4$; FPSC \& CC $-2.6 \pm 0.5$ ). Only chondral layers engineered using self-assembled BMSCs stained positive for mineral (alizarin red staining) pre-implantation (Fig. $7 a)$. No evidence of mineral accumulation was observed in chondral layers engineered using a co-culture of BMSCs and CCs. These results were confirmed by biochemical assays to determine the calcium content of the chondral layer of each construct (Fig. 7b), where a co-culture of BMSCs \& CCs was found to dramatically reduce calcium accumulation compared to BMSC only controls. Chondral layers engineered using FPSCs accumulated negligible calcium in vitro.

Post-implantation it appeared that the chondral and osseous layers of osteochondral constructs were better integrated when the chondral layer was engineered using co-cultured cells, as opposed to stem cell only groups (Fig. 8a). Macroscopically, limited calcification of the chondral layer engineered using a co-culture was observed post-implantation. $\mu \mathrm{CT}$ analysis confirmed this, showing that the high level of mineral deposition observed in the chondral layer engineered using BMSCs only was almost completely absent in the BMSC \& CC co-cultured layers (Fig. 8a). A near 7-fold reduction in calcium accumulation was observed in BMSC \& CC SA layers $(1.7 \pm 0.3 \% \mathrm{w} / \mathrm{w})$, compared to BMSC only controls $(11.4 \pm 1.3 \% \mathrm{w} / \mathrm{w})$ (Fig. 8b). Furthermore, co-culture of FPSCs \& CCs also significantly reduced calcium accumulation compared to FPSC only SA controls (reduction from $3.8 \pm 0.9 \% \mathrm{w} / \mathrm{w}$ in FPSC only to $1.0 \pm 0.5 \% \mathrm{w} / \mathrm{w}$ in co-culture). Histologically, the SA chondral layer engineered using a co-culture of 

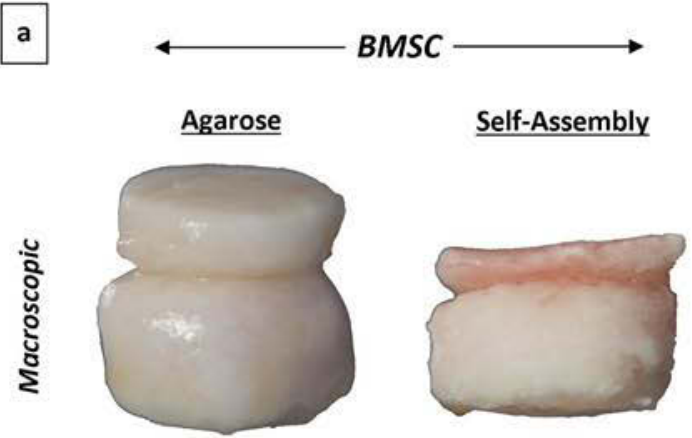

$1 \mathrm{~mm}$

છ
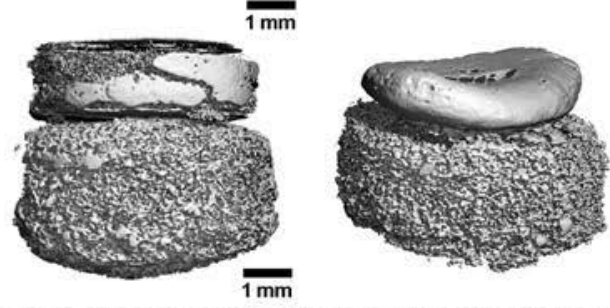
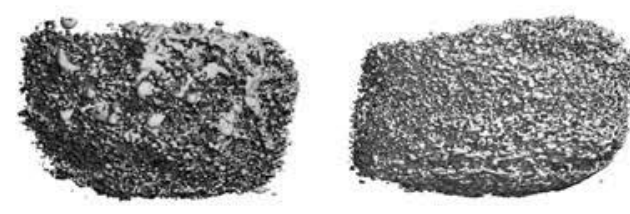

b
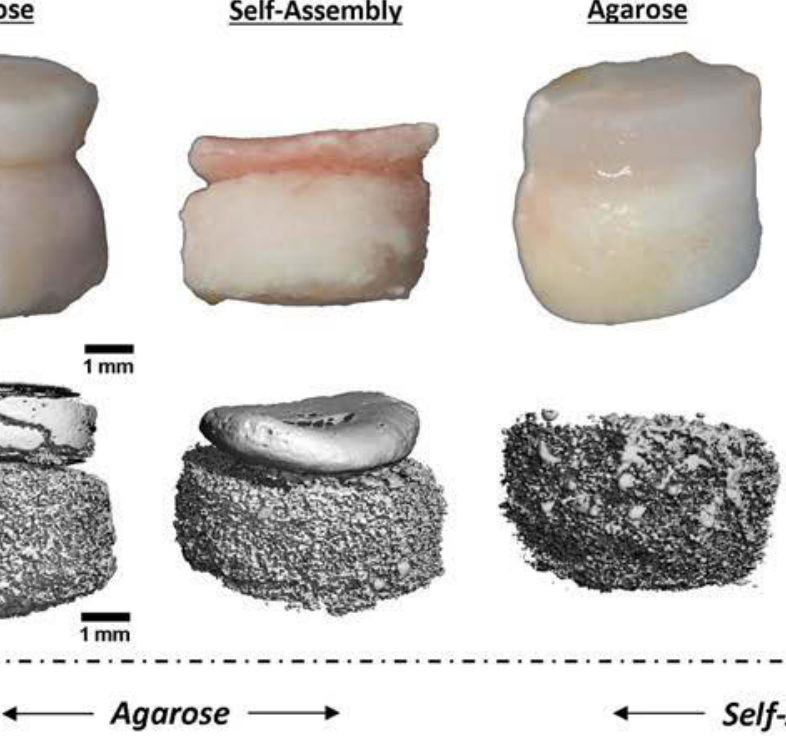

Self-Assembly
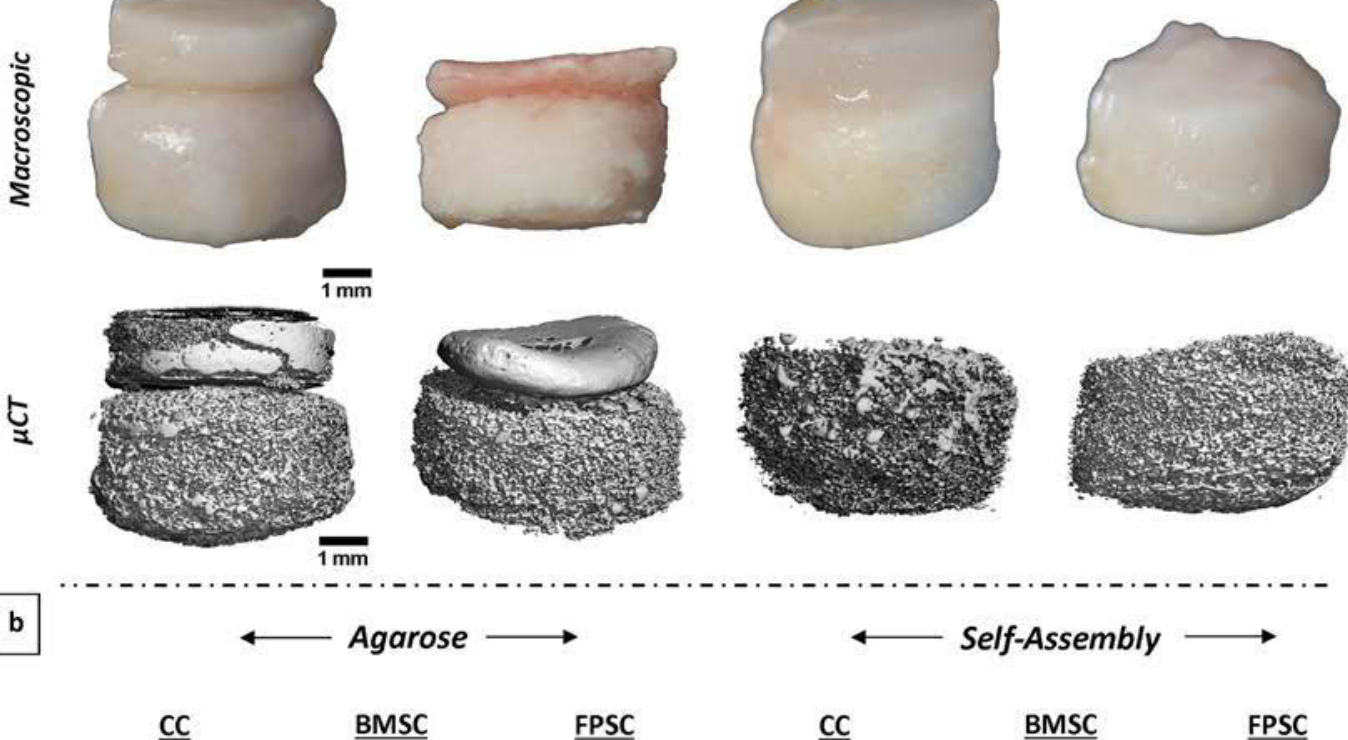

CC $\quad$ BMSC

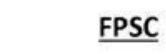

FPSC

$\underline{\mathrm{CC}}$

Self-Assembly
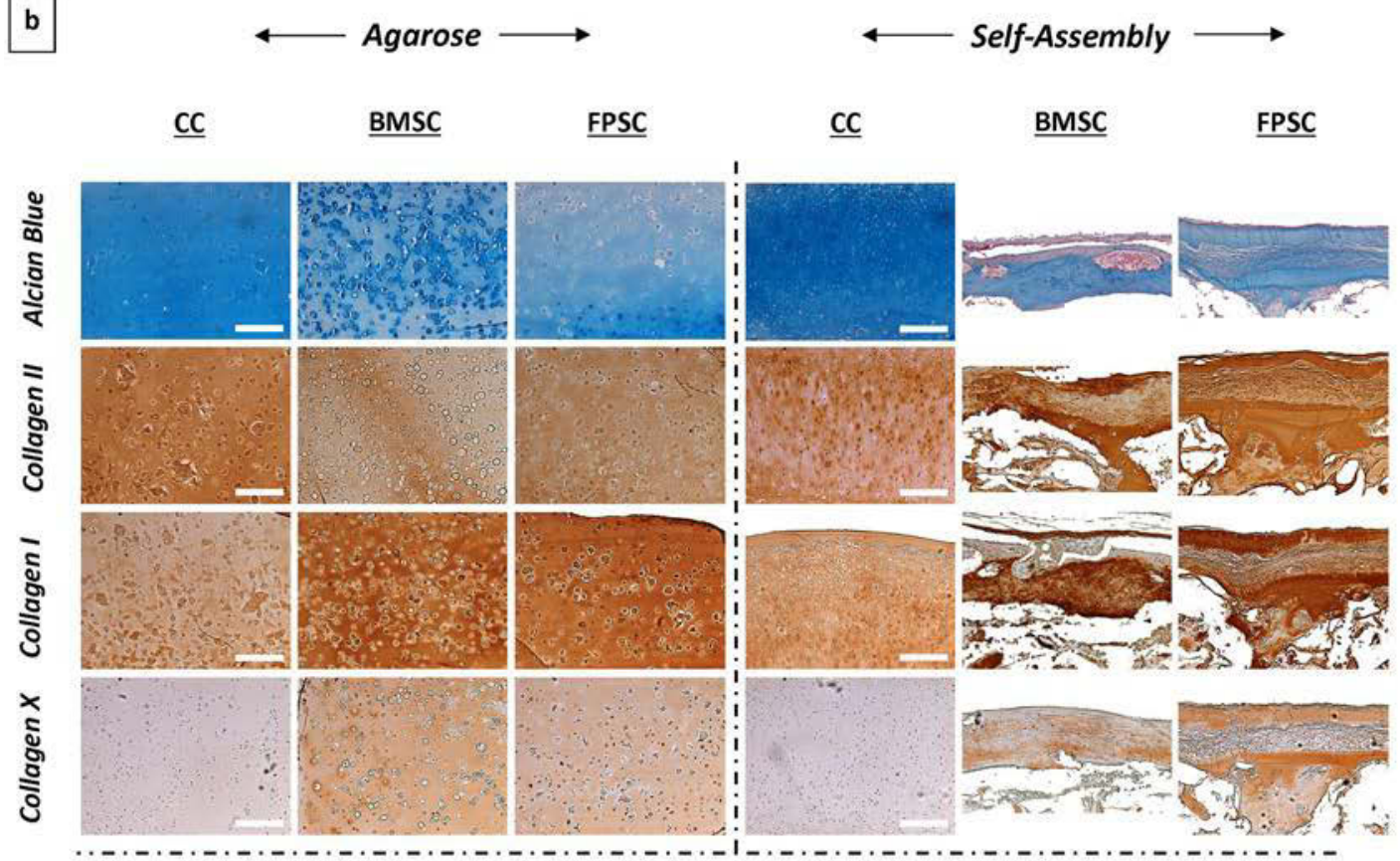

c
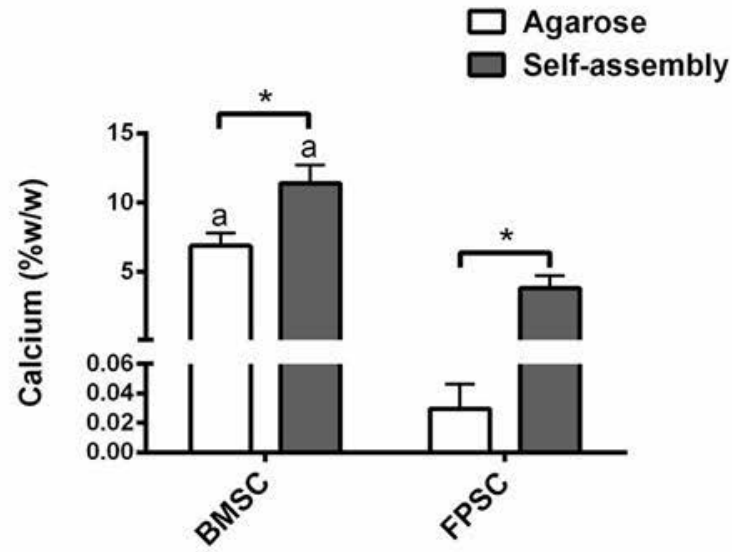

Fig. 6. Osteochondral constructs post-implantation, with chondral layers formed using either bone marrow-derived MSCs (BMSCs) or infrapatellar fat pad-derived stem cells (FPSCs). (a) Macroscopic images and $\mu \mathrm{CT}$ scans of bilayered constructs. (b) Alcian blue staining and collagen immunohistochemistry of chondral layers (chondrocyte (CC) chondral layers included for comparison purposes). (c) Calcium accumulation (\% w/w) within the chondral layers of osteochondral constructs. ${ }^{a} p<0.05$ versus corresponding FPSC group in same scaffold. ${ }^{*}$ denotes significant difference with $p<0.05$. Scale bar $=200 \mu \mathrm{m}$. 


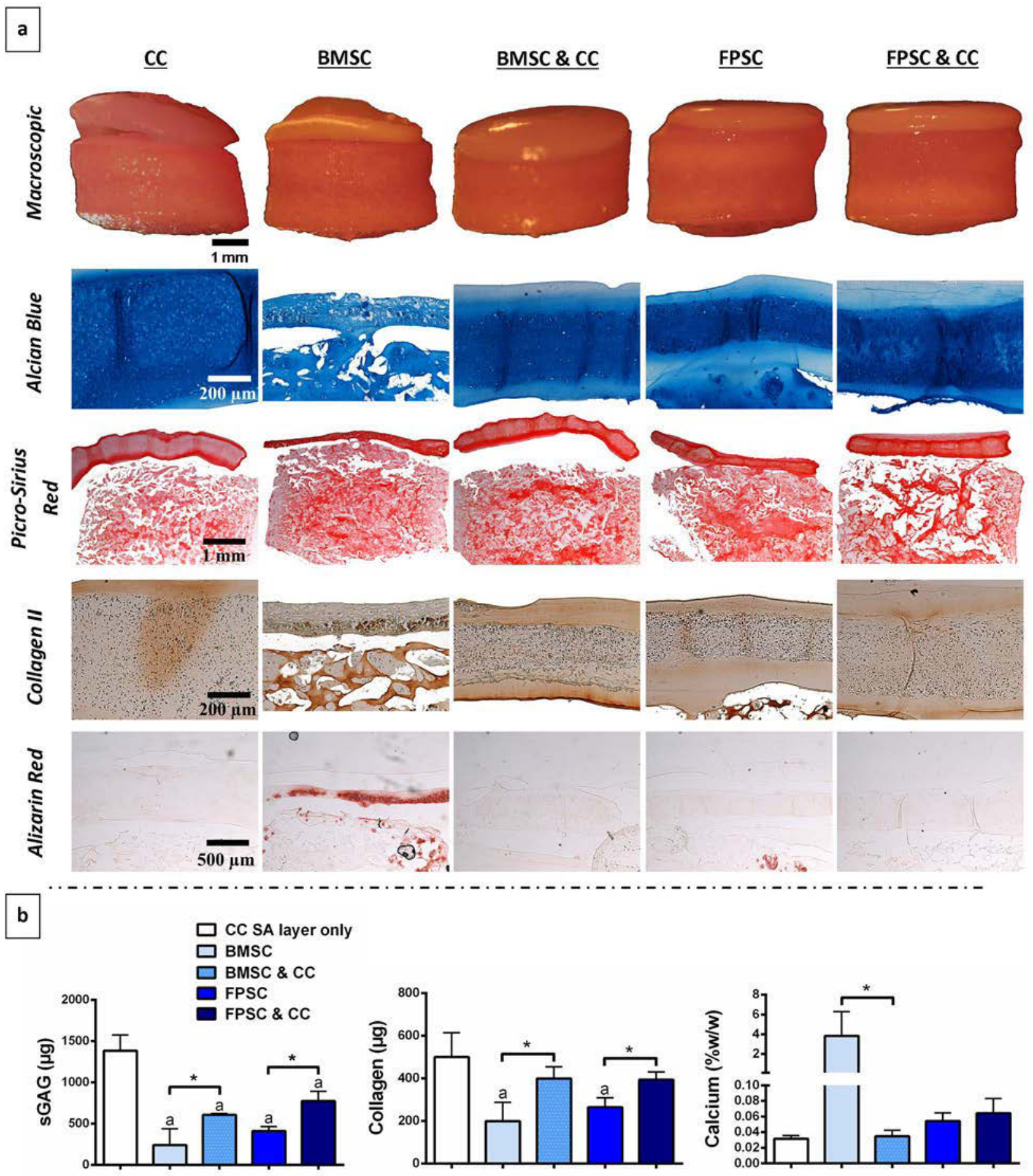

Fig. 7. Osteochondral constructs pre-implantation, with chondral layers formed through self-assembly (SA) of either chondrocytes (CCs), bone marrow-derived MSCs (BMSCs), BMSCs \& CCs, fat pad-derived stem cells (FPSCs) or FPSCs \& CCs. (a) Macroscopic images of bilayered constructs followed by chondral layer staining of alcian blue for sGAG, picro-sirius red for collagen (both chondral and osseous layer), type II collagen immunohistochemistry and alizarin red for mineralisation. (b) sGAG $(\mu \mathrm{g})$, collagen $(\mu \mathrm{g})$ and calcium $(\% \mathrm{w} / \mathrm{w})$ accumulation within SA chondral layers of osteochondral constructs. ${ }^{\mathrm{a}} p<0.05$ versus CC SA layer. * denotes significant difference with $p<0.05$.

stem cells and CCs appeared thicker, more homogeneous, more morphologically stable and more cartilage-like compared to stem cell only groups (Fig. 8a). sGAG and type II collagen staining of the chondral layers of co-culture groups was comparable to chondral layers engineered using CCs only.
This study to determine if phenotypically stable cartilage could be generated in vivo using a co-culture of CCs and either BMSCs or FPSCs was repeated, using agarose encapsulation as opposed to SA to engineer the chondral layer (Fig. 9a). Very similar results were obtained, with a co-culture of CCs and either BMSCs 
a
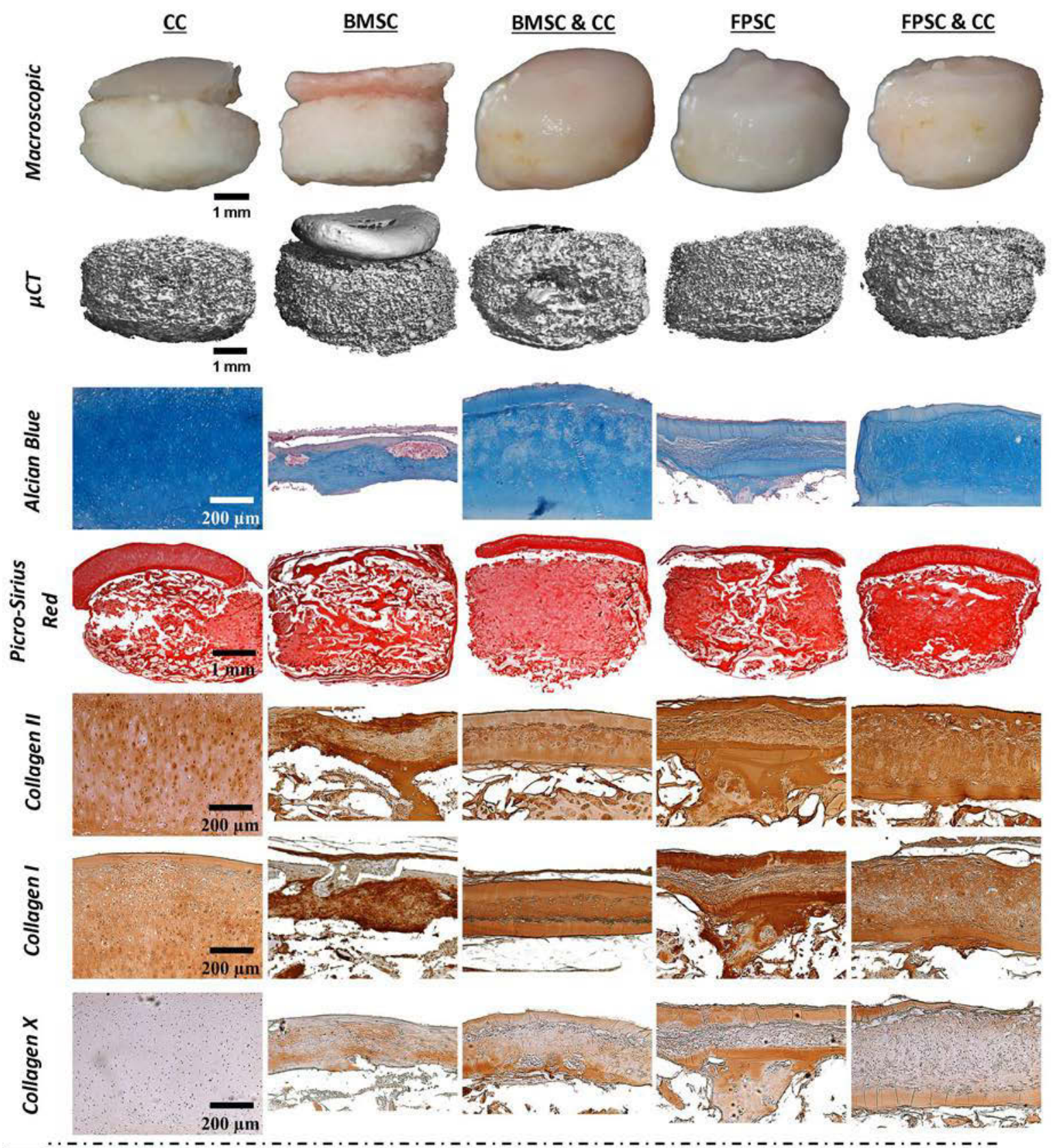

b

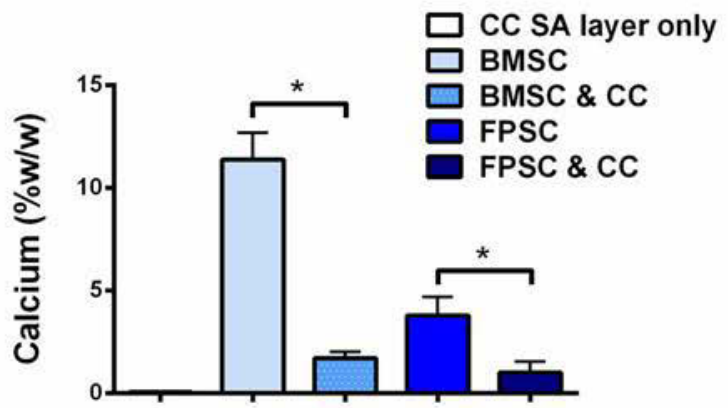

Fig. 8. Osteochondral constructs post-implantation, with chondral layers formed through self-assembly (SA) of either chondrocytes (CCs), bone marrow-derived MSCs (BMSCs), BMSCs \& CCs, fat pad-derived stem cells (FPSCs) or FPSCs \& CCs. (a) Macroscopic images of bilayered constructs followed by $\mu$ CT scans, chondral layer alcian blue staining, picro-sirius red staining (whole osteochondral construct) and collagen immunohistochemistry of chondral layers. (b) Calcium accumulation $(\% \mathrm{w} / \mathrm{w})$ within SA chondral layers of osteochondral constructs. * denotes significant difference with $p<0.05$. 


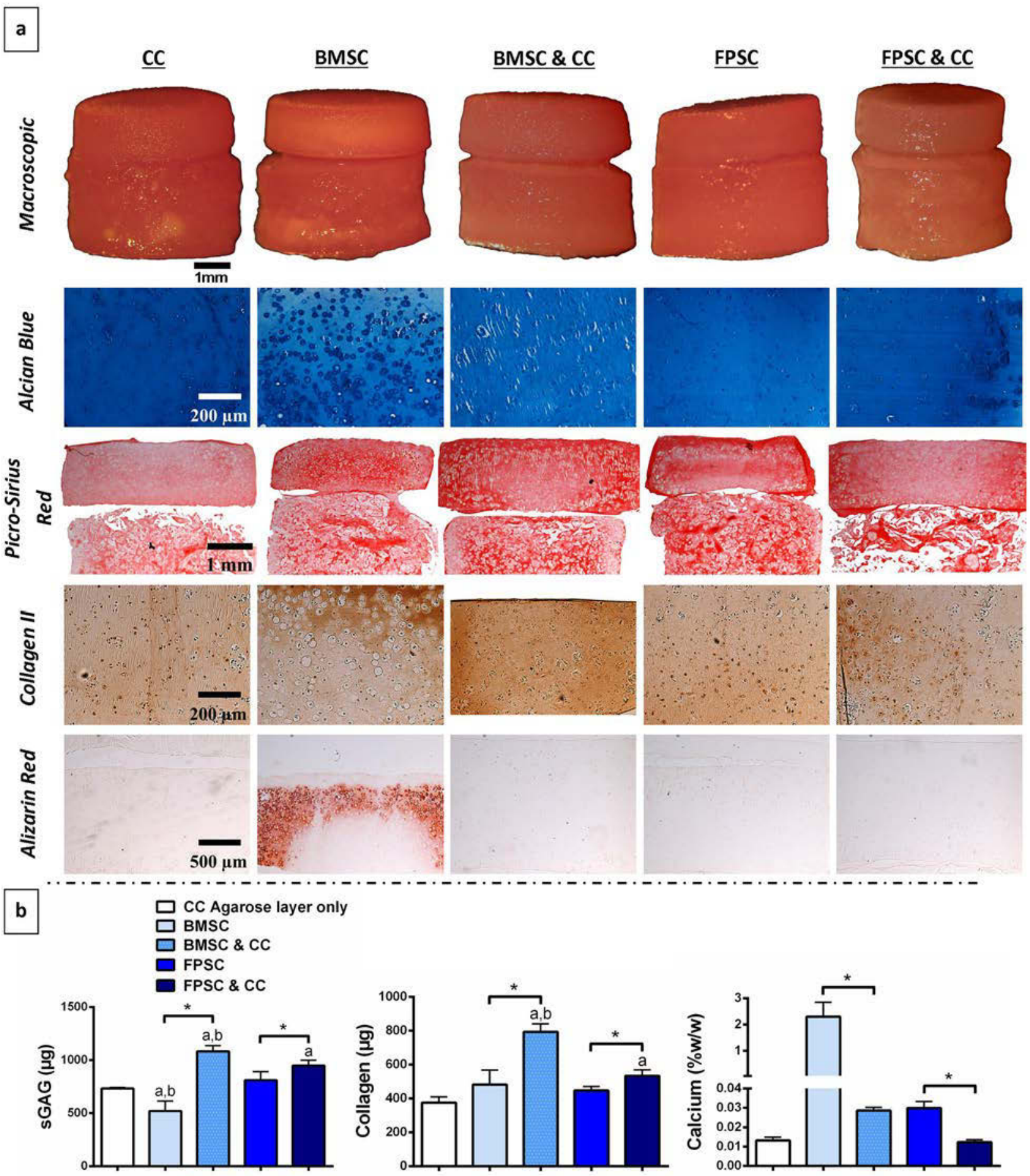

Fig. 9. Osteochondral constructs pre-implantation, with chondral layers formed through agarose encapsulation of either chondrocytes (CCs), bone marrow-derived MSCs (BMSCs), BMSCs \& CCs, fat pad-derived stem cells (FPSCs) or FPSCs \& CCs. (a) Macroscopic images of bilayered constructs followed by chondral layer staining of alcian blue for sGAG, picro-sirius red for collagen (both chondral and osseous layer), type II collagen immunohistochemistry and alizarin red for mineralisation. (b) $\mathrm{sGAG}(\mu \mathrm{g})$, collagen $(\mu \mathrm{g})$ and calcium $(\% \mathrm{w} / \mathrm{w})$ accumulation within agarose chondral layers of osteochondral constructs. ${ }^{\mathbf{a}} p<0.05$ versus $\mathrm{CC}$ seeded agarose layer. ${ }^{\mathbf{b}} p<0.05$ versus corresponding group containing FPSCs. * denotes significant difference with $p<0.05$.

or FPSCs again leading to higher levels of both sGAG and collagen accumulation pre-implantation compared to cartilage tissue-engineered using either stem cell type alone (Fig. 9b). Similar results were observed for agarose hydrogels when matrix levels were normalised to tissue wet weight $(\mathrm{sGAG}(\% \mathrm{w} / \mathrm{w})$ : CC only $-2.6 \pm 0.1$;
BMSC only $-2.2 \pm 0.4$; BMSC \& CC $-3.4 \pm 0.3$; FPSC only $-2.8 \pm 0.4$; FPSC \& $\mathrm{CC}-3.3 \pm 0.5$ and collagen (\% w/w): CC only $-1.3 \pm 0.1$; BMSC only $-2.2 \pm 0.1$; BMSC \& CC $-2.5 \pm 0.3$; FPSC only $-1.6 \pm 0.1$; FPSC $\& \mathrm{CC}-1.9 \pm 0.3)$. Post-implantation, $\mu \mathrm{CT}$ analysis demonstrated that the high level of mineral deposition 


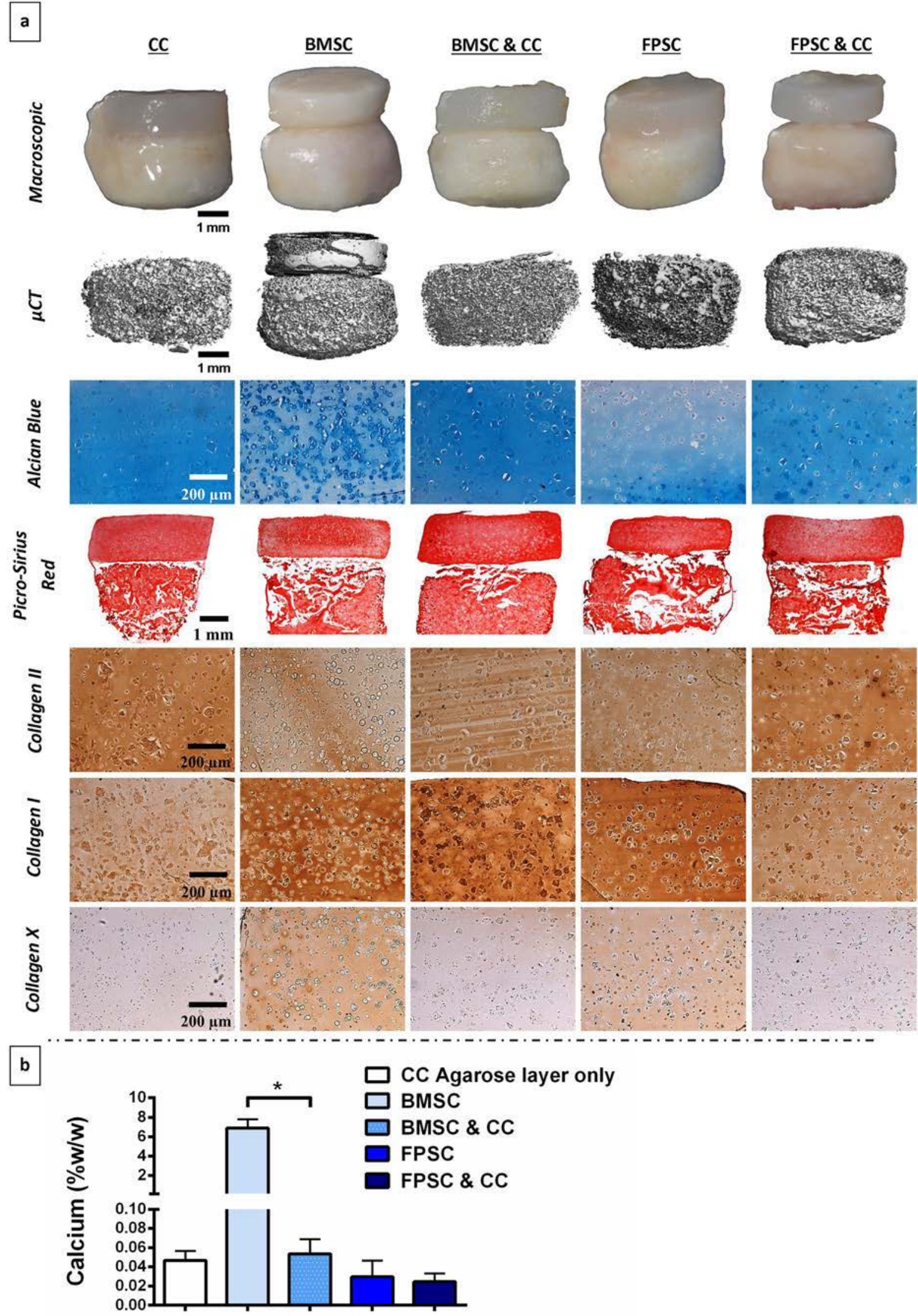

Fig. 10. Osteochondral constructs post-implantation, with chondral layers formed through agarose encapsulation of either chondrocytes (CCs), bone marrow-derived MSCs (BMSCs), BMSCs \& CCs, fat pad-derived stem cells (FPSCs) or FPSCs \& CCs. (a) Macroscopic images of bilayered constructs followed by $\mu$ CT scans, chondral layer alcian blue staining, picro-sirius red staining (whole osteochondral construct) and collagen immunohistochemistry of chondral layers. (b) Calcium accumulation $(\% \mathrm{w} / \mathrm{w})$ within agarose chondral layers of osteochondral constructs. * denotes significant difference with $p<0.05$. 


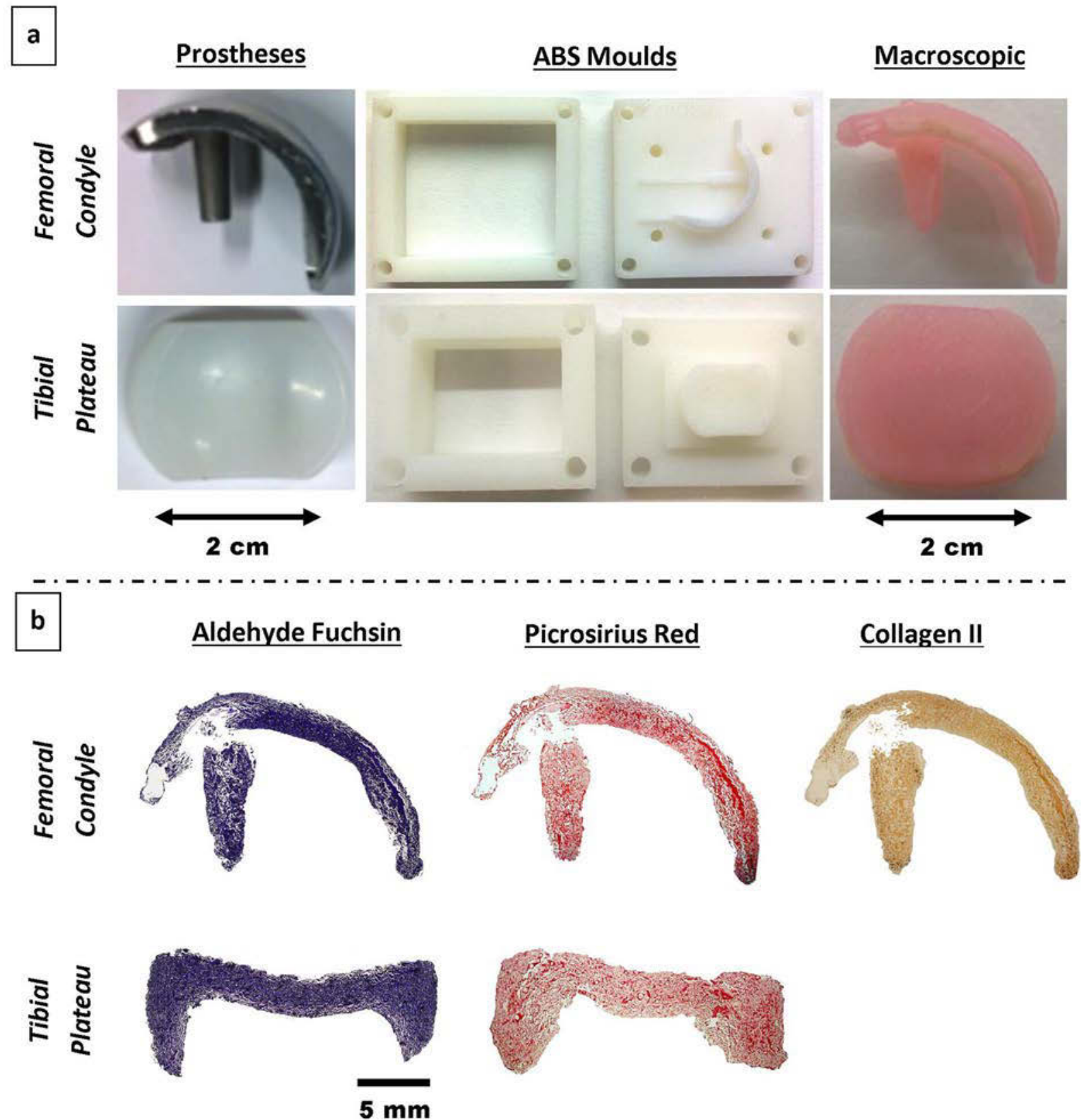

Fig. 11. Creating scaled-up, anatomically shaped alginate constructs. (a) Prostheses of the femoral condyle and tibial plateau were used to create acrylonitrile butadiene styrene (ABS) reverse moulds, from which scaled-up, anatomically shaped BMSC seeded alginate constructs were created. (b) These constructs stained positively for sGAG (aldehyde fuchsin) and type II collagen.

observed in the chondral layer engineered using BMSCs only was completely absent in the BMSC \& CC co-cultured agarose layers (Fig. 10a). Histologically, the agarose chondral layers engineered using a co-culture of stem cells and CCs appeared more cartilage-like compared to stem cell only groups, with stronger staining of sGAG and type II collagen in co-culture groups. Co-culture of stem cells and CCs was found to clearly reduce type X collagen expression in agarose chondral layers compared to stem cell only groups in vivo.
Tissue engineering a scaled-up anatomically shaped osteochondral construct for joint re-surfacing

In the final part of the study, scaled up BMSC-seeded alginate constructs ( $\sim 2 \mathrm{~cm}$ diameter) mimicking the geometry of the femoral and tibial components of a partial knee replacement prosthesis were generated from moulds fabricated by rapid prototyping (Fig. 11a). These scaled-up BMSC laden alginate constructs (in the shape of the femoral condyle and tibial plateau) were cultured in chondrogenic conditions in vitro, with the engineered 


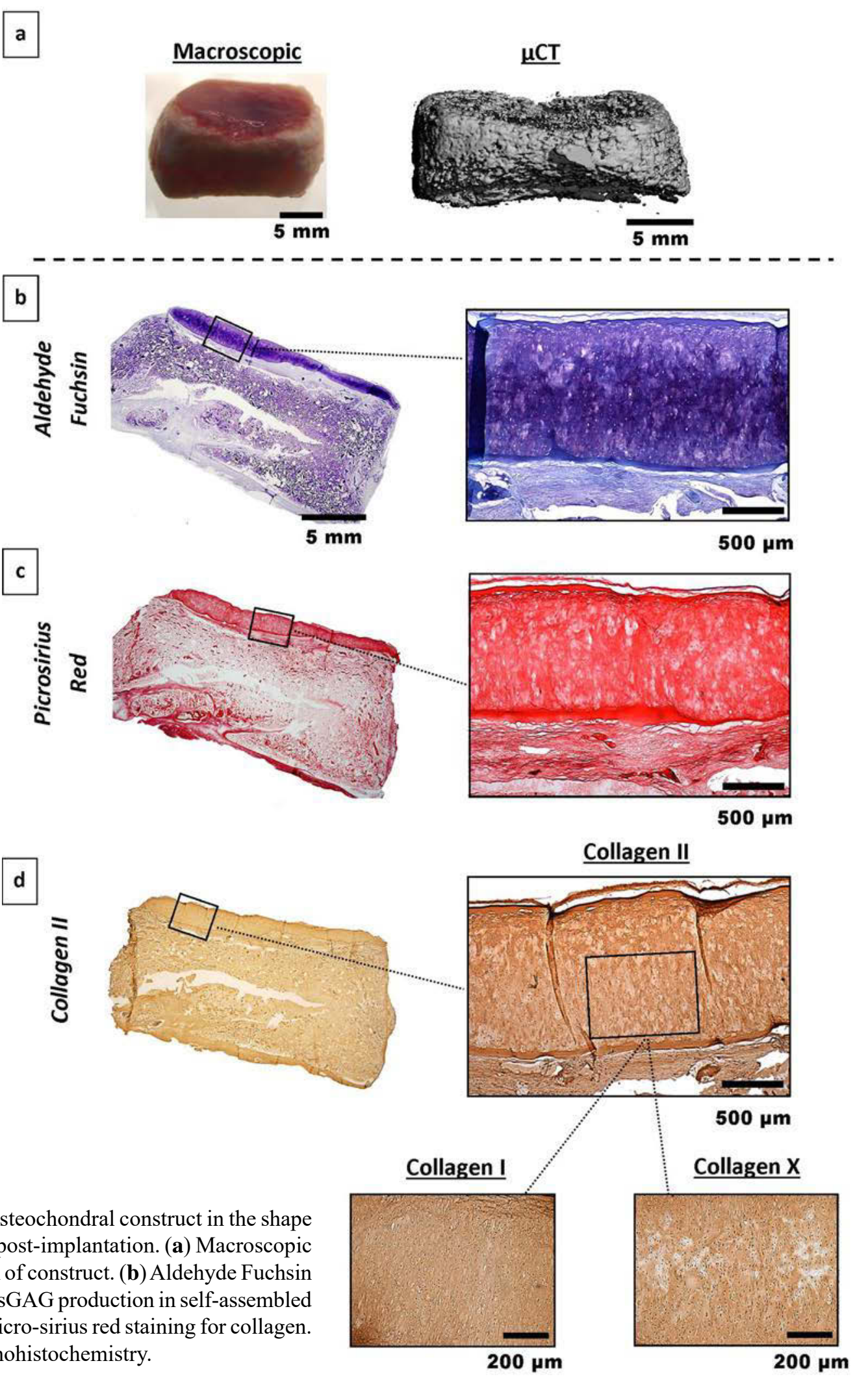

Fig. 12. Scaled-up osteochondral construct in the shape of the tibial plateau post-implantation. (a) Macroscopic image and $\mu \mathrm{CT}$ scan of construct. (b) Aldehyde Fuchsin staining with strong sGAG production in self-assembled chondral layer. (c) Picro-sirius red staining for collagen. (d) Collagen immunohistochemistry.

osteochondral constructs were implanted subcutaneously

tissues staining positively for sGAG and collagen type II after 6 weeks in culture (Fig. 11b).

Scaled-up osteochondral constructs were generated by covering the alginate hydrogel with a self-assembled layer ( $\sim 2 \mathrm{~cm}$ diameter) of engineered articular cartilage tissue (generated using a co-culture of BMSCs \& CCs in 4:1 ratio). After 6 weeks in vitro culture, the scaled-up into nude mice.

After 8 weeks in vivo a layer of cartilage remained on the top surface of the scaled-up anatomically shaped engineered implants (Fig. 12b-d), bearing a resemblance to native articular cartilage. The chondral layer of these scaled-up constructs stained strongly for sGAG (Fig. 12b) 


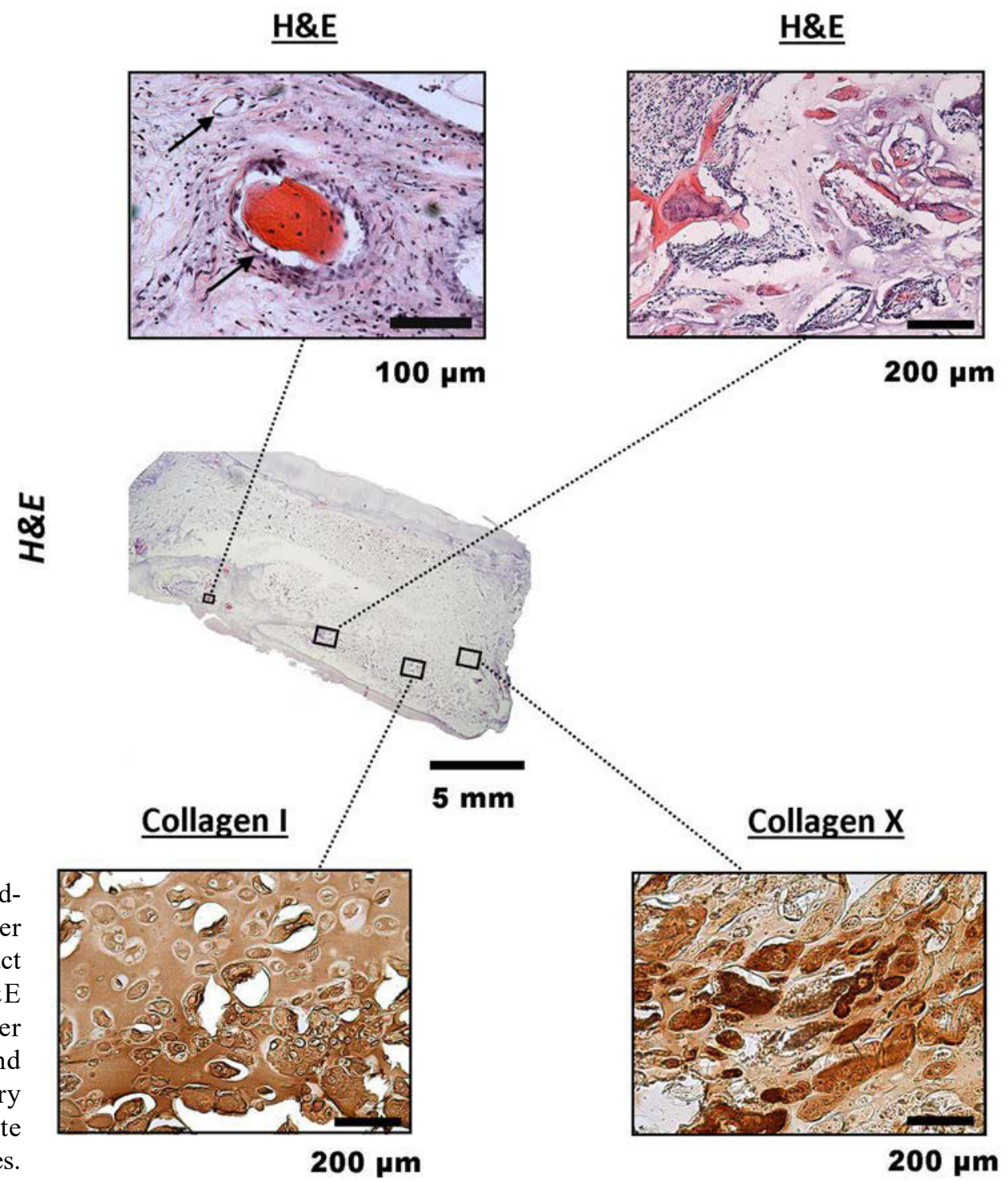

Fig. 13. Analysis of scaledup osseous alginate layer of osteochondral construct post-implantation. H\&E staining of alginate layer and collagen types I and $\mathrm{X}$ immunohistochemistry of same. Arrows indicate blood vessel-like structures.

$$
200 \mu \mathrm{m}
$$

200 pm

and type II collagen (Fig. 12d), although some evidence of type I and type X collagen deposition was also observed (Fig. 12d).

$\mu \mathrm{CT}$ analysis confirmed the deposition of mineral within the osseous region of the scaled-up constructs (Fig. 12a). H\&E staining also provided evidence of immature bone formation (Fig. 13). Blood vessel structures were detected in these H\&E stained samples. Finally, there was strong staining of collagen types I and X throughout the scaled-up BMSC laden alginate construct (Fig. 13), which would again suggest that bone was formed through the process of endochondral ossification.

\section{Discussion}

The overall aim of this study was to tissue-engineer scaledup, anatomically shaped osteochondral constructs that ultimately could be used as an alternative to traditional metal and polymer joint replacement prostheses. To that end, it was first demonstrated that a BMSC seeded alginate hydrogel (5 $\mathrm{mm}$ diameter) could support endochondral bone formation in vivo to generate the osseous layer of an osteochondral construct. Focusing next on the chondral layer, it was found that unlike chondrocytes, a phenotypically stable layer of cartilage tissue could not be generated in vivo using MSCs. This occurred irrespective of whether the chondral layer was engineered using hydrogel encapsulation or using a scaffold-free or self-assembly (SA) approach (for both BMSCs and FPSCs). Interestingly, incorporating even a small number of chondrocytes (CCs) with a larger number of MSCs (either BMSCs or FPSCs) was shown to enhance the in vitro development of the chondral layer of the constructs, and critically lead to the generation of a phenotypically stable cartilage tissue in vivo. Co-culture led to the development of thicker, more homogeneous and more morphologically stable cartilage tissues with dramatically reduced mineralisation/ calcification in vivo. In the final part of the study, scaledup BMSC-seeded alginate constructs $(\sim 2 \mathrm{~cm}$ diameter $)$ mimicking the geometry of the femoral and tibial components of a partial knee-replacement prosthesis were generated from ABS moulds fabricated by rapid prototyping. These hypertrophic cartilaginous templates 
were covered by a self-assembled layer ( $\sim 2 \mathrm{~cm}$ diameter $)$ of engineered articular cartilage (generated using a coculture of BMSCs \& CCs) to form scaled-up osteochondral constructs. After 8 weeks in vivo, a layer of cartilage remained on the surface of these scaled-up biological implants. There was also evidence of mineralisation and immature bone development in the underlying osseous layer of the engineered graft.

Two strategies were used to engineer the chondral layers of these osteochondral constructs, namely agarose encapsulation and SA. The composition of the chondral layer of osteochondral constructs engineered in vitro through SA of BMSCs approached levels seen in immature articular cartilage (Gannon et al., 2012; Mow et al., 1992). Compared to agarose encapsulation, SA using either BMSCs or FPSCs led to the generation of a more cartilage-like articular layer in vitro. In spite of this, such chondral layers engineered using stem cells alone failed to form phenotypically stable articular cartilage in vivo. In agreement with what has been observed previously (Dickhut et al., 2008; Scotti et al., 2013; Sheehy et al., 2013; Vinardell et al., 2012), cartilage tissues engineered using BMSCs appeared to proceed down the endochondral pathway in vivo, with increased type $\mathrm{X}$ collagen expression and mineralisation of the engineered tissue. This occurred for both agarose encapsulation and SA. Engineering the chondral layer using FPSCs was also problematic, as it appeared to undergo fibrous dedifferentiation in vivo, as evidenced by the in vivo development of a tissue staining less intensely for sGAG and strongly for type I collagen, with no evidence of mineralisation.

In an attempt to engineer a more phenotypically stable cartilaginous construct, we next utilised a co-culture of CCs with either BMSCs or FPSCs (MSCs to CCs in 4:1 ratio) to engineer the chondral layer of osteochondral grafts. In agreement with previous studies, a mixed co-culture of $\mathrm{CCs}$ and MSCs was found to enhance cartilage specific extracellular matrix (ECM) deposition in vitro (Acharya et al., 2012; Bian et al., 2011; Meretoja et al., 2012; Tsuchiya et al., 2004; Wu et al., 2011). Previous studies have demonstrated that this is due to MSCs secreting factors that drive proliferation of the $\mathrm{CC}$ population (Acharya et al., 2012; Wu et al., 2013; Wu et al., 2011). Co-culture led to the development of thicker, more homogeneous and more morphologically stable cartilaginous constructs in vivo (compared to corresponding stem cell only groups) that better integrated with the underlying osseous layer. In agreement with previous subcutaneous nude mouse studies (Dahlin et al., 2014; Liu et al., 2010; Sabatino et al., 2012), co-culture of CCs and stem cells also led to more robust chondrogenesis (increased sGAG and type II collagen accumulation) in the chondral layers in vivo.

In addition to enhancing the biochemical development of the chondral layer of osteochondral constructs in vitro, co-culture also appeared to suppress hypertrophy within BMSC \& CC co-cultured cartilaginous constructs, as shown by significant reductions in mineralisation of the co-cultured tissues compared to BMSC only chondral layers. Co-culture of BMSCs and CCs has previously been shown to suppress markers of BMSC hypertrophy in vitro and in vivo (Acharya et al., 2012; Bian et al., 2011;
Dahlin et al., 2014; Fischer et al., 2010; Kang et al., 2012). Remarkably, co-culture also almost completely suppressed mineralisation of the chondral layer in vivo. The most dramatic reductions in type $\mathrm{X}$ collagen deposition were observed in agarose hydrogel co-cultures, which may be due to the hypoxia promoting nature of agarose (Emans et al., 2010), as low oxygen conditions are known to suppress hypertrophy (Leijten et al., 2014; Sheehy et al., 2012). It has previously been speculated that suppression of hypertrophy is mediated, at least in part, by CCs secreting parathyroid hormone-related protein (PTHrP) (Fischer et al., 2010). In addition, a relative increase in the ratio of CCs to MSCs due to the latter cell type releasing factors that increase CC proliferation (Wu et al., 2013) would also be expected to reduce the over hypertrophic potential of the engineered tissue by increasing the ratio of phenotypically stable CCs to hypertrophic BMSCs. In addition, it has been reported that when BMSCs and CCs are co-cultured, the former cells die off over time (Meretoja et al., 2012; Wu et al., 2011), further increasing the ratio of CCs to BMSCs in the engineered tissue. Importantly, from a translational perspective, the results of this study also demonstrate that only a relatively small number of chondrocytes (4:1 ratio of MSCs to CCs) are required to suppress hypertrophy, and furthermore, that this inhibitory effect of co-culture can potentially be translated to engineer much larger cartilaginous constructs of a clinically relevant size $(\sim 2 \mathrm{~cm}$ diameter). Longer-term studies in more clinically relevant pre-clinical models are required to confirm that such cocultures truly lead to the development of phenotypically stable articular cartilage.

The final phase of the study demonstrated that it is possible to scale-up tissue-engineering approaches to generate biological osteochondral implants of a clinically relevant size. The chondral layers of these scaled-up osteochondral constructs were formed through the SA approach, as opposed to agarose encapsulation. This decision was based on in vitro data demonstrating that SA results in the formation of denser cartilaginous constructs, accumulating greater levels of sGAG and collagen than tissues engineered using agarose encapsulation. As BMSCs are essential for forming the endochondral bone layer of the osteochondral constructs, the scaled-up SA chondral layers were formed utilising BMSCs (and CCs) instead of FPSCs to minimise the number of cell sources needed in generating these osteochondral constructs. From a clinical perspective, isolating only BMSCs and CCs from a patient would be more appealing than having to isolate FPSCs, BMSCs and CCs when no clear benefit of using FPSCs over BMSCs was observed for engineering the chondral layer. Previous studies have generated scaled-up cartilaginous constructs of clinically relevant dimensions (Ding et al., 2013; Hung et al., 2003; Santoro et al., 2010), but these studies only employed chondrocytes to form the cartilage layer. As the availability of healthy CCs is limited in OA sufferers, this study importantly shows that scaled-up cartilaginous constructs can be formed using a small number of CCs mixed with a much larger number of MSCs. In addition, previous attempts to engineer scaledup endochondral bone using BMSC seeded collagen mesh scaffolds resulted in the development of a core region of 
constructs devoid of cells and matrix (Scotti et al., 2013), highlighting the importance of utilising a suitable scaffold material when engineering such large bony constructs. Future work will attempt to tailor the degradation kinetics of alginate hydrogels (through gamma-irradiation) to accelerate its degradation in vivo (Alsberg et al., 2003; Simmons et al., 2004) in the hope that this in turn will facilitate more rapid endochondral bone formation in the appropriate regions of scaled up grafts.

The geometry of the osseous region of the scaled-up implants was formed by injecting BMSC laden alginate into moulds created using scans taken of existing joint replacement prostheses. There are a number of different approaches proposed in the literature to form the osseous region of anatomically accurate osteochondral constructs. For example, devitalised trabecular bone disks in the shape of a human patella have been used (Hung et al., 2003). Osteogenically primed BMSC-seeded poly- $\varepsilon$ caprolactone/hydroxyapatite composites have been used to form the osseous layer of osteochondral constructs mimicking both the proximal tibial joint condyle (Lee et al., 2009) and the femoral condyle (Ding et al., 2013). Osteogenically primed BMSC-seeded poly (ethylene glycol)-based hydrogels have also been used (Alhadlaq et al., 2004). In this study, the osseous layer of the scaledup osteochondral constructs was developed through endochondral ossification of chondrogenically primed BMSCs. In theory, the advantage of exploiting the endochondral route for engineering such scaled-up grafts is that CCs are inherently capable of surviving the low oxygen, nutrient deprived conditions that would exist in such scaled-up anatomic constructs. Developmentally, all long bones are derived from a cartilaginous pre-cursor, making recapitulation of such processes an appealing route to bone regeneration. The moulding technique used in this study is transferable to any geometry, suggesting that this approach could be used in the regeneration of any joint. Another key question that needs to be addressed with such endochondral tissue-engineering strategies is whether in vivo subcutaneous maturation of such a construct would be required prior to implantation into a load bearing orthotopic environment, or whether it would be possible to implant the in vitro engineered cartilaginous graft directly into the defect site (and allow endochondral ossification to occur orthotopically). Previous studies have proposed using an ectopic environment as an in vivo bioreactor (Emans et al., 2010; Stevens et al., 2005) to allow maturation of an engineered tissue, with the resulting tissue subsequently implanted into an orthotopic defect site. If such a strategy is not adopted, it may be necessary to mechanically reinforce the osseous region of the osteochondral implant prior to implantation into a high load-bearing environment such as the knee. We are currently exploring the use of 3D printing technologies to tissue-engineer such mechanically reinforced, tissue-engineered grafts for endochondral bone and osteochondral defect regeneration.

\section{Conclusions}

The present study provides a framework for tissueengineering biological joint replacement prostheses for regenerating damaged/diseased joints. Clearly a number of challenges remain, including confirmation of efficacy of this approach within a load bearing orthotopic environment and implementation of this approach using diseased human MSCs and CCs. However, if these challenges can be overcome, it may lead to the development of a novel tissueengineered therapy for the millions of people suffering from OA worldwide.

\section{Acknowledgements}

Funding was provided by the Irish Research Council for Science, Engineering and Technology (G30403), the SFI President of Ireland Young Researcher Award (08/Y15/ B1336), and a European Research Council Starter Grant (StemRepair-Project number: 258463). We would like to acknowledge the contribution of Simon McCoy in creating the presented ABS moulds through rapid prototyping techniques.

\section{Disclosure Statement}

No conflicts of interest exist.

\section{References}

Acharya C, Adesida A, Zajac P, Mumme M, Riesle J, Martin I, Barbero A (2012) Enhanced chondrocyte proliferation and mesenchymal stromal cells chondrogenesis in coculture pellets mediate improved cartilage formation. J Cell Physiol 227: 88-97.

Alhadlaq A, Elisseeff JH, Hong L, Williams CG, Caplan AI, Sharma B, Kopher RA, Tomkoria S, Lennon DP, Lopez A, Mao JJ (2004) Adult stem cell driven genesis of humanshaped articular condyle. Ann Biomed Eng 32: 911-923.

Alsberg E, Kong HJ, Hirano Y, Smith MK, Albeiruti A, Mooney DJ (2003) Regulating bone formation via controlled scaffold degradation. J Dent Res 82: 903-908.

Benya PD, Shaffer JD (1982) Dedifferentiated chondrocytes reexpress the differentiated collagen phenotype when cultured in agarose gels. Cell 30: 215-224.

Bian L, Zhai DY, Mauck RL, Burdick JA (2011) Coculture of human mesenchymal stem cells and articular chondrocytes reduces hypertrophy and enhances functional properties of engineered cartilage. Tissue Eng Part A 17: 1137-1145.

Brittberg M, Lindahl A, Nilsson A, Ohlsson C, Isaksson O, Peterson L (1994) Treatment of deep cartilage defects in the knee with autologous chondrocyte transplantation. N Engl J Med 331: 889-895.

Buckley CT, Kelly DJ (2012) Expansion in the presence of FGF-2 enhances the functional development of cartilaginous tissues engineered using infrapatellar fat pad derived MSCs. J Mech Behav Biomed Mater 11: 102-111. 
Chen J, Chen H, Li P, Diao H, Zhu S, Dong L, Wang R, Guo T, Zhao J, Zhang J (2011) Simultaneous regeneration of articular cartilage and subchondral bone in vivo using MSCs induced by a spatially controlled gene delivery system in bilayered integrated scaffolds. Biomaterials 32 4793-4805.

Dahlin RL, Kinard LA, Lam J, Needham CJ, Lu S, Kasper FK, Mikos AG (2014) Articular chondrocytes and mesenchymal stem cells seeded on biodegradable scaffolds for the repair of cartilage in a rat osteochondral defect model. Biomaterials 35: 7460-7469.

Diaz-Romero J, Gaillard JP, Grogan SP, Nesic D, Trub T, Mainil-Varlet P (2005) Immunophenotypic analysis of human articular chondrocytes: changes in surface markers associated with cell expansion in monolayer culture. J Cell Physiol 202: 731-742.

Dickhut A, Gottwald E, Steck E, Heisel C, Richter W (2008) Chondrogenesis of mesenchymal stem cells in gel-like biomaterials in vitro and in vivo. Front Biosci 13: 4517-4528.

Ding C, Qiao Z, Jiang W, Li H, Wei J, Zhou G, Dai K (2013) Regeneration of a goat femoral head using a tissue-specific, biphasic scaffold fabricated with CAD/ CAM technology. Biomaterials 34: 6706-6716.

Emans PJ, van Rhijn LW, Welting TJ, Cremers A, Wijnands N, Spaapen F, Voncken JW, Shastri VP (2010) Autologous engineering of cartilage. Proc Natl Acad Sci USA 107: 3418-3423.

EnobakhareBO, BaderDL, LeeDA(1996)Quantification of sulfated glycosaminoglycans in chondrocyte/alginate cultures, by use of 1,9-dimethylmethylene blue. Anal Biochem 243: 189-191.

Farrell E, Both SK, Odorfer KI, Koevoet W, Kops N, O’Brien FJ, Baatenburg de Jong RJ, Verhaar JA, Cuijpers V, Jansen J, Erben RG, van Osch GJ (2011) In-vivo generation of bone via endochondral ossification by in-vitro chondrogenic priming of adult human and rat mesenchymal stem cells. BMC Musculoskelet Disord 12: 31.

Farrell E, van der Jagt OP, Koevoet W, Kops N, van Manen CJ, Hellingman CA, Jahr H, O’Brien FJ, Verhaar JA, Weinans H, van Osch GJ (2009) Chondrogenic priming of human bone marrow stromal cells: a better route to bone repair? Tissue Eng Part C Methods 15: 285-295.

Fischer J, Dickhut A, Rickert M, Richter W (2010) Human articular chondrocytes secrete parathyroid hormonerelated protein and inhibit hypertrophy of mesenchymal stem cells in coculture during chondrogenesis. Arthritis Rheum 62: 2696-2706.

Gannon AR, Nagel T, Kelly DJ (2012) The role of the superficial region in determining the dynamic properties of articular cartilage. Osteoarthritis Cartilage 20: 1417-1425.

Grayson WL, Bhumiratana S, Grace Chao PH, Hung CT, Vunjak-Novakovic G (2010) Spatial regulation of human mesenchymal stem cell differentiation in engineered osteochondral constructs: effects of pre-differentiation, soluble factors and medium perfusion. Osteoarthritis Cartilage 18: 714-723.

Guilak F (2010) Homing in on a biological joint replacement. Stem Cell Res Ther 1: 40.

Harada N, Watanabe Y, Sato K, Abe S, Yamanaka K, Sakai Y, Kaneko T, Matsushita T (2014) Bone regeneration in a massive rat femur defect through endochondral ossification achieved with chondrogenically differentiated MSCs in a degradable scaffold. Biomaterials 35: 78007810 .

Hayes DA, Miller LE, Block JE (2012) Knee Osteoarthritis Treatment with the KineSpring Knee Implant System: A Report of Two Cases. Case Rep Orthop 2012: 297326.

Hung CT, Lima EG, Mauck RL, Takai E, LeRoux MA, Lu HH, Stark RG, Guo XE, Ateshian GA (2003) Anatomically shaped osteochondral constructs for articular cartilage repair. J Biomech 36: 1853-1864.

Igarashi T, Iwasaki N, Kasahara Y, Minami A (2010) A cellular implantation system using an injectable ultrapurified alginate gel for repair of osteochondral defects in a rabbit model. J Biomed Mater Res A 94: 844-855.

Ignat'eva NY, Danilov NA, Averkiev SV, Obrezkova MV, Lunin VV, Sobol EN (2007) Determination of hydroxyproline in tissues and the evaluation of the collagen content of the tissues. Zhurnal Analiticheskoi Khimii 62: 51-57.

Janicki P, Kasten P, Kleinschmidt K, Luginbuehl R, Richter W (2010) Chondrogenic pre-induction of human mesenchymal stem cells on $\beta$-TCP: enhanced bone quality by endochondral heterotopic bone formation. Acta Biomater 6: 3292-3301.

Kafienah W, Sims TJ (2004) Biochemical methods for the analysis of tissue-engineered cartilage. Methods Mol Biol 238: 217-230.

Kang N, Liu X, Guan Y, Wang J, Gong F, Yang X, Yan L, Wang Q, Fu X, Cao Y, Xiao R (2012) Effects of coculturing BMSCs and auricular chondrocytes on the elastic modulus and hypertrophy of tissue engineered cartilage. Biomaterials 33: 4535-4544.

Keeney JA, Eunice S, Pashos G, Wright RW, Clohisy JC (2011) What is the evidence for total knee arthroplasty in young patients?: A systematic review of the literature. Clin Orthop Relat Res 469: 574-583.

Kim YJ, Sah RL, Doong JY, Grodzinsky AJ (1988) Fluorometric assay of DNA in cartilage explants using Hoechst 33258. Anal Biochem 174: 168-176.

Kock L, Van Donkelaar CC, Ito K (2012) Tissue engineering of functional articular cartilage: The current status. Cell Tissue Res 347: 613-627.

Kolambkar YM, Boerckel JD, Dupont KM, Bajin M, Huebsch N, Mooney DJ, Hutmacher DW, Guldberg RE (2011a) Spatiotemporal delivery of bone morphogenetic protein enhances functional repair of segmental bone defects. Bone 49: 485-492.

Kolambkar YM, Dupont KM, Boerckel JD, Huebsch N, Mooney DJ, Hutmacher DW, Guldberg RE (2011b) An alginate-based hybrid system for growth factor delivery in the functional repair of large bone defects. Biomaterials 32: 65-74.

Kurtz SM, Lau E, Ong K, Zhao K, Kelly M, Bozic KJ (2009) Future young patient demand for primary and revision joint replacement: National projections from 2010 to 2030. Clin Orthop Relat Res 467: 2606-2612.

Lee CH, Cook JL, Mendelson A, Moioli EK, Yao H, Mao JJ (2010) Regeneration of the articular surface of the 
rabbit synovial joint by cell homing: A proof of concept study. Lancet 376: 440-448.

Lee CH, Marion NW, Hollister S, Mao JJ (2009) Tissue formation and vascularization in anatomically shaped human joint condyle ectopically in vivo. Tissue Eng Part A 15: 3923-3930.

Lee KY, Mooney DJ (2012) Alginate: Properties and biomedical applications. Prog Polym Sci (Oxford) 37: 106-126.

Leijten J, Georgi N, Moreira Teixeira L, van Blitterswijk CA, Post JN, Karperien M (2014) Metabolic programming of mesenchymal stromal cells by oxygen tension directs chondrogenic cell fate. Proc Natl Acad Sci U S A 111: 13954-13959.

Lennon DP, Caplan AI (2006) Isolation of human marrow-derived mesenchymal stem cells. Exp Hematol 34: 1604-1605.

Liu X, Sun H, Yan D, Zhang L, Lv X, Liu T, Zhang W, Liu W, Cao Y, Zhou G (2010) In vivo ectopic chondrogenesis of BMSCs directed by mature chondrocytes. Biomaterials 31: 9406-9414.

Liu Y, Buckley CT, Almeida HV, Mulhall KJ, Kelly DJ (2014) Infrapatellar fat pad-derived stem cells maintain their chondrogenic capacity in disease and can be used to engineer cartilaginous grafts of clinically relevant dimensions. Tissue Eng Part A 20: 3050-3062.

Ma HM, Lu YC, Ho FY, Huang CH (2005) Long-term results of total condylar knee arthroplasty. J Arthroplasty 20: $580-584$.

Mano JF, Reis RL (2007) Osteochondral defects: Present situation and tissue engineering approaches. J Tissue Eng Regen Med 1: 261-273.

Martin I, Miot S, Barbero A, Jakob M, Wendt D (2007) Osteochondral tissue engineering. J Biomech 40: 750-765.

Mason JM, Grande DA, Barcia M, Grant R, Pergolizzi RG, Breitbart AS (1998) Expression of human bone morphogenic protein 7 in primary rabbit periosteal cells: Potential utility in gene therapy for osteochondral repair. Gene Ther 5: 1098-1104.

Meretoja VV, Dahlin RL, Kasper FK, Mikos AG (2012) Enhanced chondrogenesis in co-cultures with articular chondrocytes and mesenchymal stem cells. Biomaterials 33: 6362-6369.

Mesallati T, Buckley CT, Kelly DJ (2014a) A comparison of self-assembly and hydrogel encapsulation as a means to engineer functional cartilaginous grafts using culture expanded chondrocytes. Tissue Eng Part C Methods 20: $52-63$.

Mesallati T, Buckley CT, Kelly DJ (2014b) Engineering articular cartilage-like grafts by self-assembly of infrapatellar fat pad-derived stem cells. Biotechnol Bioeng 111: $1686-1698$.

Mesallati T, Buckley CT, Nagel T, Kelly DJ (2013) Scaffold architecture determines chondrocyte response to externally applied dynamic compression. Biomech Model Mechanobiol 12: 889-899.

Mow VC, Ratcliffe A, Poole AR (1992) Cartilage and diarthrodial joints as paradigms for hierarchical materials and structures. Biomaterials 13: 67-97.
Pavone V, Boettner F, Fickert S, Sculco TP (2001) Total condylar knee arthroplasty: A long-term followup. Clin Orthop Relat Res: 18-25.

Pittenger MF, Mackay AM, Beck SC, Jaiswal RK, Douglas R, Mosca JD, Moorman MA, Simonetti DW, Craig S, Marshak DR (1999) Multilineage potential of adult human mesenchymal stem cells. Science 284: 143-147.

Rodrigues MT, Lee SJ, Gomes ME, Reis RL, Atala A, Yoo JJ (2012) Bilayered constructs aimed at osteochondral strategies: The influence of medium supplements in the osteogenic and chondrogenic differentiation of amniotic fluid-derived stem cells. Acta Biomater 8: 2795-2806.

Sabatino MA, Santoro R, Gueven S, Jaquiery C, Wendt DJ, Martin I, Moretti M, Barbero A (2012) Cartilage graft engineering by co-culturing primary human articular chondrocytes with human bone marrow stromal cells. J Tissue Eng Regen Med, in press.

Santo VE, Gomes ME, Mano JF, Reis RL (2013) Controlled release strategies for bone, cartilage, and osteochondral engineering-part i: Recapitulation of native tissue healing and variables for the design of delivery systems. Tissue Eng Part B Rev 19: 308-326.

Santoro R, Olivares AL, Brans G, Wirz D, Longinotti C, Lacroix D, Martin I, Wendt D (2010) Bioreactor based engineering of large-scale human cartilage grafts for joint resurfacing. Biomaterials 31: 8946-8952.

Scotti C, Piccinini E, Takizawa H, Todorov A, Bourgine P, Papadimitropoulos A, Barbero A, Manz MG, Martin I (2013) Engineering of a functional bone organ through endochondral ossification. Proc Natl Acad Sci USA 110: 3997-4002.

Scotti C, Tonnarelli B, Papadimitropoulos A, Scherberich A, Schaeren S, Schauerte A, Lopez-Rios J, Zeller R, Barbero A, Martin I (2010) Recapitulation of endochondral bone formation using human adult mesenchymal stem cells as a paradigm for developmental engineering. Proc Natl Acad Sci U S A 107: 7251-7256.

Segawa Y, Muneta T, Makino H, Nimura A, Mochizuki T, Ju YJ, Ezura Y, Umezawa A, Sekiya I (2009) Mesenchymal stem cells derived from synovium, meniscus, anterior cruciate ligament, and articular chondrocytes share similar gene expression profiles. J Orthop Res 27: 435-441.

Seil R, Pape D (2011) Causes of failure and etiology of painful primary total knee arthroplasty. Knee Surg Sports Traumatol Arthrosc 19: 1418-1432.

Sheehy EJ, Buckley CT, Kelly DJ (2012) Oxygen tension regulates the osteogenic, chondrogenic and endochondral phenotype of bone marrow derived mesenchymal stem cells. Biochem Biophys Res Commun 417: 305-310.

Sheehy EJ, Vinardell T, Buckley CT, Kelly DJ (2013) Engineering osteochondral constructs through spatial regulation of endochondral ossification. Acta Biomater 9: 5484-5492.

Sheehy EJ, Vinardell T, Toner ME, Buckley CT, Kelly DJ (2014) Altering the architecture of tissue engineered hypertrophic cartilaginous grafts facilitates vascularisation and accelerates mineralisation. PLoS One 9: e90716.

Shen B, Wei A, Tao H, Diwan AD, Ma DD (2009) BMP2 enhances TGF- $\beta 3$-mediated chondrogenic differentiation of human bone marrow multipotent mesenchymal stromal 
cells in alginate bead culture. Tissue Eng Part A 15: 13111320 .

Simmons CA, Alsberg E, Hsiong S, Kim WJ, Mooney DJ (2004) Dual growth factor delivery and controlled scaffold degradation enhance in vivo bone formation by transplanted bone marrow stromal cells. Bone 35: 562-569.

Stevens MM, Marini RP, Schaefer D, Aronson J, Langer $\mathrm{R}$, Shastri VP (2005) In vivo engineering of organs: the bone bioreactor. Proc Natl Acad Sci U S A 102: 11450 11455.

Temenoff JS, Mikos AG (2000) Review: Tissue engineering for regeneration of articular cartilage. Biomaterials 21: 431-440.

Tsuchiya K, Chen G, Ushida T, Matsuno T, Tateishi $\mathrm{T}$ (2004) The effect of coculture of chondrocytes with mesenchymal stem cells on their cartilaginous phenotype in vitro. Mater Sci Eng C Mater Biol Appl 24: 391-396.

Van der Kraan PM, Van den Berg WB (2012) Chondrocyte hypertrophy and osteoarthritis: Role in initiation and progression of cartilage degeneration? Osteoarthritis Cartilage 20: 223-232.

Van der Stok J, Koolen MK, Jahr H, Kops N, Waarsing JH, Weinans H, Van der Jagt OP (2014) Chondrogenically differentiated mesenchymal stromal cell pellets stimulate endochondral bone regeneration in critical-sized bone defects. Eur Cell Mater 27: 137-148.

Vinardell T, Sheehy EJ, Buckley CT, Kelly DJ (2012) A comparison of the functionality and in vivo phenotypic stability of cartilaginous tissues engineered from different stem cell sources. Tissue Eng Part A 18: 1161-1170.

Wang X, Grogan SP, Rieser F, Winkelmann V, Maquet V, La Berge M, Mainil-Varlet P (2004) Tissue engineering of biphasic cartilage constructs using various biodegradable scaffolds: An in vitro study. Biomaterials 25: 3681-3688.

Wendt D, Jakob M, Martin I (2005) Bioreactor-based engineering of osteochondral grafts: from model systems to tissue manufacturing. J Biosci Bioeng 100: 489-494.

Woolf AD, Pfleger B (2003) Burden of major musculoskeletal conditions. Bull World Health Organ 81: 646-656.

Wu L, Leijten J, Van Blitterswijk CA, Karperien M (2013) Fibroblast growth factor-1 is a mesenchymal stromal cell-secreted factor stimulating proliferation of osteoarthritic chondrocytes in co-culture. Stem Cells Dev 22: 2356-2367.

Wu L, Leijten JCH, Georgi N, Post JN, Van Blitterswijk CA, Karperien M (2011) Trophic effects of mesenchymal stem cells increase chondrocyte proliferation and matrix formation. Tissue Eng Part A 17: 1425-1436.

\section{Discussion with Reviewers}

Reviewer I: How was the SA chondral layer attached on top of the anatomically shaped osseous layer? The SA layer should have a planar structure and some level of rigidity. Is any glue or crosslinking used to ensure the good matching of these two layers? This was not clearly explained in the methods section.

Authors: The self-assembled chondral layer was attached to the top of the anatomically shaped alginate layer using a small amount of fibrin gel (to act like glue) to aid initial integration. This fibrin gel was applied sparingly around the perimeter of the self-assembled construct before attachment.

Reviewer I: Mechanical property of the tissue-engineered cartilage is critical to the success after implantation. Can the authors comment on how the mechanical properties of the bilayer construct, especially the anatomically shaped construct, can be further improved?

Authors: The mechanical properties of the anatomically shaped osseous construct could be improved by attempting to reinforce the alginate hydrogel base before implantation into a load-bearing environment. One option for this would be to utilise 3D bioprinting technologies to first create a "skeleton" of polycaprolactone (PCL) fibres (or another biocompatible polymer) forming the outline of an anatomically shaped construct, and to then fill this scaledup PCL scaffold with a MSC laden alginate hydrogel using layer-by-layer deposition (Kundu et al., 2013, additional reference; Schuurman et al., 2011, additional reference). The alginate would be allowed to cross-link in the presence of calcium chloride or similar. These PCL reinforced alginate constructs would be better able to withstand much larger initial forces in vivo. The mechanical properties of the bilayered constructs could also be improved through the use of bioreactors and/or channelled architectures throughout the constructs, with such technologies possibly increasing nutrient transfer and enhancing cartilage specific matrix synthesis within the engineered tissues (Bian et al., 2009, additional reference; Buckley et al., 2009, additional reference; Haasper et al., 2008, additional reference; Kelly et al., 2004, additional reference; Martin et al., 2004, additional reference; Mauck et al., 2000, additional reference; $\mathrm{Ng}$ et al., 2006, additional reference; VunjakNovakovic et al., 1999, additional reference; Wendt et al., 2005, text reference).

Reviewer I: Can the authors discuss potential strategies to improve the integration strength between the osseous and chondral layers?

Authors: 3D printing a single unifying "skeleton" of polycaprolactone (PCL) fibres (or similar), which was common to both the osseous and chondral layers, could help improve the integration strength. In addition, alginate could be covalently modified with RGD-containing peptides to control cell behaviour (Simmons et al., 2004, text reference). Such a modification could be expected to lead to enhanced vascularisation and/or endochondral bone formation in vivo, with enhanced bone formation possibly leading to greater integration between the chondral and osseous layers.

\section{Additional References}

Bian L, Angione SL, Ng KW, Lima EG, Williams DY, Mao DQ, Ateshian GA, Hung CT (2009) Influence of decreasing nutrient path length on the development of engineered cartilage. Osteoarthritis Cartilage 17: 677-685. 
Buckley CT, Thorpe SD, Kelly DJ (2009) Engineering of large cartilaginous tissues through the use of microchanneled hydrogels and rotational culture. Tissue Eng Part A 15: 3213-3220.

Haasper C, Zeichen J, Meister R, Krettek C, Jagodzinski M (2008) Tissue engineering of osteochondral constructs in vitro using bioreactors. Injury 39: 66-76.

Kelly TAN, Wang CCB, Mauck RL, Ateshian GA, Hung CT (2004) Role of cell-associated matrix in the development of free-swelling and dynamically loaded chondrocyte-seeded agarose gels. Biorheology 41: 223237.

Kundu J, Shim JH, Jang J, Kim SW, Cho DW (2013) An additive manufacturing-based PCL-alginate-chondrocyte bioprinted scaffold for cartilage tissue engineering. J Tissue Eng Regen Med, doi: 10.1002/term.1682.

Martin I, Wendt D, Heberer M (2004) The role of bioreactors in tissue engineering. Trends Biotechnol 22: 80-86.

Mauck RL, Soltz MA, Wang CC, Wong DD, Chao PH, Valhmu WB, Hung CT, Ateshian GA (2000) Functional tissue engineering of articular cartilage through dynamic loading of chondrocyte-seeded agarose gels. J Biomech Eng 122: 252-260.

$\mathrm{Ng} \mathrm{KW}$, Mauck RL, Statman LY, Lin EY, Ateshian GA, Hung CT (2006) Dynamic deformational loading results in selective application of mechanical stimulation in a layered, tissue-engineered cartilage construct. Biorheology 43: 497-507.

Schuurman W, Khristov V, Pot MW, van Weeren PR, Dhert WJ, Malda J (2011) Bioprinting of hybrid tissue constructs with tailorable mechanical properties. Biofabrication 3: 021001.

Vunjak-Novakovic G, Martin I, Obradovic B, Treppo S, Grodzinsky AJ, Langer R, Freed LE (1999) Bioreactor cultivation conditions modulate the composition and mechanical properties of tissue-engineered cartilage. $\mathrm{J}$ Orthop Res 17: 130-138.

Editor's Note: Scientific Editor in charge of the paper: Martin Stoddart. 